\title{
Nationwide cohort study on the epidemiology and survival outcomes of thyroid cancer
}

\author{
Fu-Chao Liu ${ }^{1,2, *}$, Huan-Tang Lin ${ }^{1,2, *}$, Shu-Fu Lin ${ }^{3}$, Chang-Fu Kuo ${ }^{4,5}$, Ting-Ting Chung ${ }^{6}$ \\ and Huang-Ping $\mathbf{Y} \mathbf{u}^{1,2}$ \\ ${ }^{1}$ Department of Anesthesiology, Chang Gung Memorial Hospital, Taoyuan, Taiwan \\ ${ }^{2}$ College of Medicine, Chang Gung University, Taoyuan, Taiwan \\ ${ }^{3}$ Department of Endocrinology, Chang Gung Memorial Hospital, Taoyuan, Taiwan \\ ${ }^{4}$ Division of Rheumatology, Allergy and Immunology, Chang Gung Memorial Hospital, Taoyuan, Taiwan \\ ${ }^{5}$ Division of Rheumatology, Orthopaedics and Dermatology, University of Nottingham, Nottingham, UK \\ ${ }^{6}$ Office for Big Data Research, Chang Gung Memorial Hospital, Taoyuan, Taiwan \\ *These authors have contributed equally in this study \\ Correspondence to: Huang-Ping Yu, email: yuhp2001@adm.cgmh.org.tw \\ Keywords: thyroid cancer, epidemiology, survival outcome, National Health Insurance, Taiwan Cancer Registry \\ Received: April 28, $2017 \quad$ Accepted: May 31, $2017 \quad$ Published: July 22, 2017 \\ Copyright: Liu et al. This is an open-access article distributed under the terms of the Creative Commons Attribution License 3.0 \\ (CC BY 3.0), which permits unrestricted use, distribution, and reproduction in any medium, provided the original author and source \\ are credited.
}

\section{ABSTRACT}

In the past three decades, the thyroid cancer incidence has surged globally. Herein, the Taiwan National Health Insurance database was used to identify thyroid cancer patients and to estimate the prevalence and incidence of thyroid cancer during 1997-2012. The Taiwan Cancer Registry and the National Death Registry databases were crosslinked to obtain information on the histological subtypes and survival rates. Joinpoint regression analysis was used for estimating the average annual percentage changes (APCs) in prevalence, incidence, and survival. The agestandardized incidence of thyroid cancer increased from 5.66 per 100,000 personyears in 1997 to 12.30 per 100,000 person-years in 2012, with an average APC of 5.1 (6.9 in males, 4.6 in females). Thyroid cancer was more prevalent in patients with high socioeconomic status and in urban areas. Papillary carcinoma was the most abundant subtype, with a 2.9-fold increase of incident cases noted during 19982012 (from $\mathbf{8 0 . 6 \%}$ to $\mathbf{8 9 . 8 \%}$ of all cases). Among the different treatments, partial thyroidectomy increased the most (average APC, 17.3). The overall survival rates by sex and subtype remained stable over time, with 5-year survival rates of $90.2 \%$ in 1997 and $92.4 \%$ in 2010 . In conclusion, 2.2- and 4.2-fold increases in the incidence and prevalence of thyroid cancer, respectively, were observed during 1997-2012 in Taiwan. The surging incidence of thyroid cancer but stable survival rates, and mainly increased in the papillary subtype, altogether imply enhanced detection of subclinical lesions. A true increase due to environmental carcinogens might also be responsible, but warrant further investigations.

\section{INTRODUCTION}

Thyroid cancer is the most common endocrine malignancy [1]. According to GLOBOCAN 2012, thyroid cancer accounted for $2.1 \%$ of the total cancer incidence in 2012, with a worldwide age-standardized incidence rate of 4.0 (1.9 in males, 6.1 in females) per 100,000 person-years and a worldwide mortality rate of $0.5(0.3$ in males, 0.6 in females) per 100,000 persons in 2012 [2]. While the incidences of most other common solid tumors 
in developed countries are either stable or decreasing, the incidence of thyroid cancer is surging in both sexes worldwide, largely coincident with the introduction of neck ultrasonography in the 1980s. Generally, patients with thyroid cancer have a favorable prognosis, with the 10-year survival rate estimated to be greater than $90 \%$ [1].

Thyroid cancer is a heterogeneous disease with distinct epidemiological and prognostic features for different histological subtypes. Thyroid follicular cells give rise to two major groups of thyroid cancers: differentiated (papillary and follicular) and undifferentiated (anaplastic, poorly differentiated) carcinomas [3]. Papillary and follicular thyroid carcinomas are more prevalent and have a relatively favorable prognosis, while the poorlydifferentiated and anaplastic subtypes are much more rare and aggressive. Papillary thyroid carcinoma is the most abundant subtype, comprising $85-90 \%$ of all thyroid cancers. Most papillary thyroid carcinomas are clinically indolent, consistent with a simple genome and low mutational densities. Papillary thyroid microadenomas, defined as tumors $<1 \mathrm{~cm}$ in size, are the most commonly diagnosed thyroid cancers and are associated with longterm disease-free survival rates of $>90 \%$ [4]. On the other hand, follicular thyroid carcinomas account for only $2-5 \%$ of thyroid cancers [3]. The follicular subtype is more aggressive than papillary carcinoma, mainly because it can metastasize via vascular invasion. Accordingly, it often presents with metastasis at the time of diagnosis.

Medullary thyroid carcinomas account for less than $5 \%$ of thyroid cancers, although they are responsible for approximately $13 \%$ of all thyroid cancer-related deaths [5]. Medullary thyroid carcinomas arise from neural crestderived parafollicular C-cells, which do not accumulate radioiodine. These cells also secrete calcitonin and carcinoembryonic antigen, which are used as specific tumor markers. Most medullary thyroid carcinomas are sporadic, and usually develop in the fourth to sixth decade of life; however, up to $25 \%$ of cases are associated with a hereditary autosomal-dominant syndrome, known as multiple endocrine neoplasia type 2. Surgical excision is the primary curative therapy for patients with medullary thyroid carcinoma.

Anaplastic thyroid carcinomas account for approximately $1-2 \%$ of thyroid cancers and are the most aggressive subtype, associated with a mean overall survival of only 6 months, and more than $80 \%$ patients die within one year of diagnosis [3]. These highly invasive tumors should be surgically removed thoroughly and treated by adjuvant locoregional radiation therapy and chemotherapy.

Epidemiologic studies have reported increasing incidences of thyroid cancer worldwide in the last three decades, with improvements in the detection of thyroid cancer considered as the main reason underlying this rising trend [1]. The additional diagnoses from screening are mainly papillary thyroid carcinomas, which, as mentioned above, are a relatively prevalent histological finding with excellent prognosis in the general population and which are generally not considered a deadly disease $[1,6]$. In fact, autopsy studies have revealed that at least one-third of adults without symptoms during their lifetime harbored small papillary thyroid carcinomas [1]. The other main explanation of the rising incidence of thyroid cancer is a true increase of thyroid carcinogenesis, driven by a higher exposure of potential risk factors such as radiation exposure, diabetes, excess weight, and/or environmental factors [1].

There are currently limited data regarding thyroid cancer epidemiology in Taiwan, and worldwide population-based cancer registries of the World Health Organization often have incomplete data of Taiwan. Previous reports from the Taiwan Cancer Registry database revealed that the age-standardized incidence of thyroid cancer increased from 1.44 per 100,000 personyears in 1980 to 13.3 per 100,000 person-years in 2012 , as the second most increased solid cancer (in both sexes), with an average annual percentage change (APC) of 6.4 $[7,8]$. However, information about the thyroid cancer subtypes and survival rates were not provided in that study. Additionally, estimating mortality rates based on the Taiwan National Health Insurance (NHI) database would result in overestimation of the true situations, because retraction from the NHI system does not always represent death. Therefore, we conducted this populationbased epidemiologic study, mainly based on the NHI database and crosslinked with the Taiwan Cancer Registry and National Death Registry databases to improve the accuracy and reliability, with the aims to estimate the secular trends of the epidemiology and survival rates of thyroid cancer in Taiwan between 1997 and 2012.

\section{RESULTS}

\section{Demographic data and geographic variations of patients with thyroid cancer}

The eligible population in our study comprised of 21,952,273 registered NHI beneficiaries (49.33\% male, $50.67 \%$ female) in 2012 in Taiwan. Of these, 25,711 patients (5,552 males and 20,160 females) with a thyroid cancer diagnosis during 1997-2012 were identified. The baseline characteristics of these thyroid cancer patients are presented in Table 1. Male thyroid cancer patients were diagnosed at an older mean age $(49.83 \pm 15.80$ years vs. $46.42 \pm 14.83$ years) and had a significantly higher Charlson Comorbidity Index (CCI) score than female patients $(2.66 \pm 2.95$ vs. $1.90 \pm 2.51)$. The majority of thyroid cancer patients lived in urban areas (63.45\%), while patients residing in rural areas comprised only $7.32 \%$ of cases. Furthermore, thyroid cancer patients more frequently had a professional occupation and high income level (quintiles 4 and 5, total 40.18\%). These results of the socioeconomic status analysis indicated that 
thyroid cancer was more prevalent in high-income urban areas in Taiwan.

Comparison based on calendar year between 1997 and 2012 revealed that the incident cases of thyroid cancer increased from 944 in 1997 to 2,656 in 2012, while the average CCI score decreased from $1.68 \pm 2.32$ to $0.71 \pm 1.88$. The female-to-male ratio of incident cases decreased from 4.65 in 1997 to 3.68 in 2012, whereas the average age of the thyroid cancer patients increased from $45.22 \pm 16.10$ to $48.66 \pm 14.74$ years, possibly due to the effect of an increased percentage of male cases $(17.69 \%$ in $1997,22.55 \%$ in 2012). The observed phenomenon of a higher prevalence in individuals with high socioeconomic status was even more pronounced when comparing the distribution in 2012 to that in 1997.

The geographic variations in thyroid cancer prevalence and incidence in Taiwan are shown in Figure 1. The regions with higher prevalence were located in the urban and industrial areas, especially in Kaohsiung and Tainan, while the prevalence was relatively low in rural and mountainous areas. No obvious correlations between thyroid cancer prevalence and the sites of nuclear power plants in Taiwan was noted.

\section{Prevalence and incidence of thyroid cancer between 1997 and 2012}

Tables 2 and 3 show the temporal trends in thyroid cancer prevalence and incidence in Taiwan during 19972012. The age-standardized prevalence of thyroid cancer was 26.2 (95\% confidence interval: 25.43-27.03) per 100,000 persons in 1997 and 109.9 (108.49-111.26) per 100,000 persons in 2012 . Joinpoint analysis of the thyroid cancer prevalence (Table 4 ) revealed that the average APC was $10.0(9.5-10.5, \mathrm{p}<0.05)$, with female patients [average APC, 10.3(9.8-10.7)] accumulating slightly faster than male cases [average APC, 9.9(9.4-10.3)]. On the other hand, the age-standardized incidence was 5.66 (5.29-6.04) per 100,000 person-years in 1997 and 12.30 (11.84-12.77) per 100,000 person-years in 2012 , with the female-to-male ratio of age-standardized incidence decreasing from 4.8 in 1997 to 3.3 in 2012 (Figure 2). Joinpoint analysis of thyroid cancer incidence (Table 5) established 2 joinpoints, with an average APC of 5.1 (2.5-7.8, $\mathrm{p}<0.05)$. In detail, the average APCs of segments 1997-1999, 1999-2004, and 2004-2012 were 12.3 (-5.9 to 34.0$),-1.1$ ( -5.9 to 4.1 ), and 7.4 (5.9 to 8.9)], respectively, with male patients showing a faster rise in incidence [average APC, 6.9(3.3-10.6)] than female cases [average APC, 4.6 (1.8-7.5)]. Overall, the standardized prevalence and incidence of thyroid cancer were 4.2-fold and 2.2-fold higher in 2012 than in 1997, respectively.

The age-specific prevalence and incidence of thyroid cancer are shown in Figure 3; the male thyroid cancer patients (peak incidence 60-69 years) were generally older than the female cases (peak incidence 50-59 years).

\section{Incidences and survival rates of different histological subtypes of thyroid cancer}

We obtained information on the different thyroid cancer subtypes from the Taiwan Cancer Registry database. Table 6 shows the demographic data of patients according to their different subtypes. Patients with the papillary subtype had the youngest age at diagnosis $(46.23 \pm 14.23$ years), contained the highest proportion of females $(79.11 \%)$, had the least comorbidities (CCI: $0.72 \pm 1.65$ ), and the highest percentages of residence in urban areas, high income levels, and professional occupations. On the other hand, patients with the anaplastic subtype presented at the oldest age ( $70.58 \pm 11.74$ years), had the most comorbidities (CCI: $2.07 \pm 2.76$ ), and were the least likely to live in urban areas and to be classified as high socioeconomic status.

Figure 4 illustrates the incidence trends of different thyroid cancer subtypes in Taiwan between 1998 and 2012. Papillary thyroid carcinoma was the most abundant subtype with the most rapid surge in incidence, growing from $80.6 \%$ of the total cases in 1998 (904 incident cases) to $89.8 \%$ in 2012 (2,601 incident cases) (Figure 5). The percentage of the other histological subtypes gradually decreased from 1998 to 2012 (follicular subtype $9.9 \%$ to $6.4 \%$, medullary subtype $2.2 \%$ to $1 \%$, anaplastic subtype $1.7 \%$ to $0.7 \%$, and other subtypes $5.5 \%$ to $2.1 \%$ ) despite the actual numbers of incident cases of the follicular subtypes increasing 1.6-fold during this period. The papillary-to-follicular incidence ratio grew from 8.14 in 1998 to 14.13 in 2012.

Although the incident percentages of the different subtypes changed significantly during this period, their survival rates were essentially stable. Joinpoint analysis of the survival rates for different subtypes (Table 7) showed no statistically significant changes during 1997-2012, with 5 -year survival rates in 2010 of $95.5 \%, 89.3 \%, 80.6 \%$, $36.4 \%$, and $7.4 \%$ for the papillary subtype, follicular subtype, medullary subtype, other subtypes, and anaplastic subtype, respectively. Owing to the accelerating incidence rate and more favorable survival, the papillary subtype has become increasingly predominant over time.

\section{Treatments for thyroid cancer}

We calculated and compared the numbers of different treatment procedures performed after the diagnosis of thyroid cancer. Among the entire cohort in Taiwan during 1998-2011, 86\% of thyroid cancer patients in Taiwan received partial or total thyroidectomy, and $61 \%$ received radioiodine ablation, while $15 \%$ of patients received no treatment at all after diagnosis (Table 8). Analysis of the thyroid cancer treatments by sex revealed that male thyroid cancer patients received more advanced treatments than female cases, including neck dissection, chemotherapy, and external beam radiotherapy. This result might be due to 
male patients having a worse prognosis than females, thus requiring more adjuvant therapies. Joinpoint analysis of the treatment procedures showed generally rising trends during 1998-2011, owing to the increase in incident cases, except for external beam radiotherapy [average APC, -2.54 (-6.91 to 2.04)] (Table 9). The most sharply accelerating trend was noted for the partial thyroidectomy, which showed an 11.5-fold increase from 1998 to 2011, with an average APC of 17.3 (14.2 to $20.5, \mathrm{p}<0.05)$. Comparing partial to total thyroidectomy, partial thyroidectomy accounted for $35.6 \%$ of thyroidectomies in 2011 but only $11.5 \%$ in 1998. The average APCs of major treatments such as total thyroidectomy [8.39 (5.93 to 10.9), p<0.05] and I-131 ablation [6.32 (4.09 to 8.59), $\mathrm{p}<0.05]$ even outpaced the average APC of thyroid cancer incidence during the period. Figure 6 shows the secular trends of different treatment procedures of thyroid cancer in Taiwan from 1997 to 2011.

\section{Analysis of thyroid cancer survival rates by sex and Charlson Comorbidity Index}

The 1-, 2-, and 5-year thyroid cancer survival rates in Taiwan were generally stable or mildly improved (Figure 7), with slightly increased average APCs during the study period (Table 10). The latest calculated 1-, 2-, and 5-year survival rates were as follows: $97.1 \%(94.5 \%$ in males, $97.9 \%$ in females) in 2012, $96.1 \%$ (92.4\% in males, $97.3 \%$ in females) in 2012, and $92.4 \%$ (84.9\% in males, $94.8 \%$ in females) in 2010, respectively. Though male thyroid cancer patients showed a relatively poor prognosis compared to female cases, their survival rate improved (5year survival rate $84.5 \%$ in $1997,84.9 \%$ in 2010$)$, with an average APC of $0.66(0.00-1.32 ; \mathrm{p}<0.05)$.

In view of the potentially significant impact of comorbidities on overall survival, we next divided our study populations into 2 groups according to their CCI score. As a result, the $\mathrm{CCI} \leq 3$ group ( $88.5 \%$ of thyroid cancer patients) showed slightly better survival rates than the overall study population, while the $\mathrm{CCI} \geq 4$ group $(11.5 \%)$ showed much lower survival rates (1-, 2-, and 5-year survival rates: $83.6 \%, 78.6 \%, 66.4 \%$, respectively) (Figure 8 ).

\section{DISCUSSION}

\section{Interpretation of current results}

In our present population-based cohort study combining data from the Taiwan NHI database, the Taiwan Cancer Registry, and the National Death Registry, the reported age-standardized incidence rate
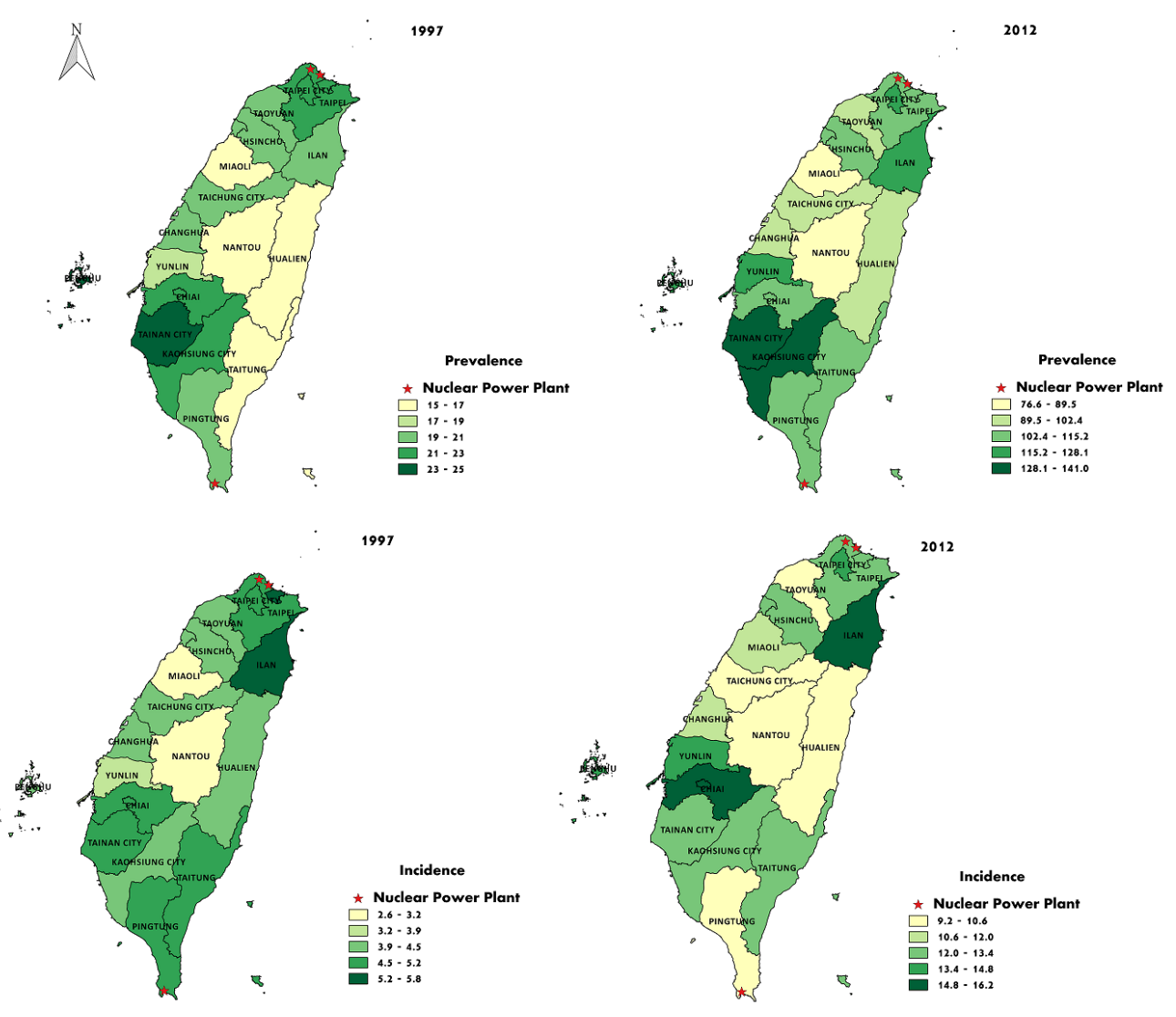

Figure 1: Geographic variations in the prevalence and incidence of thyroid cancer in Taiwan in 1997 and 2012 , and their associations with the sites of nuclear power plants in Taiwan. 
Table 1: Clinical characteristics of patients with thyroid cancer from 1997 to 2012

\begin{tabular}{|c|c|c|c|c|c|c|c|}
\hline & \multirow{2}{*}{$\begin{array}{c}\text { Entire } \\
\text { cohort } \\
(n=25,711)\end{array}$} & \multicolumn{3}{|c|}{ By sex } & \multicolumn{3}{|c|}{ By calendar year } \\
\hline & & $\begin{array}{c}\text { Female } \\
(n=20,160)\end{array}$ & $\begin{array}{c}\text { Male } \\
(n=5,551)\end{array}$ & $p$-Value & $\begin{array}{c}1997 \\
(n=994)\end{array}$ & $\begin{array}{c}2012 \\
(n=2,656)\end{array}$ & $p$-Value \\
\hline $\begin{array}{l}\text { Age (yrs) (mean } \pm \\
\text { standard deviation) }\end{array}$ & $47.46 \pm 15.11$ & $46.42 \pm 14.83$ & $49.83 \pm 15.80$ & $<.0001 *$ & $45.22 \pm 16.10$ & $48.66 \pm 14.74$ & $<.0001 *$ \\
\hline \multicolumn{8}{|l|}{ Sex, No. $(\%)$} \\
\hline Female & $\begin{array}{l}20,160 \\
(78.41)\end{array}$ & -- -- & ---- & -- & $777(82.31)$ & $\begin{array}{c}2,057 \\
(77.45)\end{array}$ & $0.0017 *$ \\
\hline Male & $\begin{array}{c}5,551 \\
(21.59)\end{array}$ & -- -- & -- -- & & $167(17.69)$ & $559(22.55)$ & \\
\hline \multicolumn{8}{|l|}{ Resident area, No. (\%) } \\
\hline Urban & $\begin{array}{l}16,313 \\
(63.45)\end{array}$ & $\begin{array}{l}12,916 \\
(64.07)\end{array}$ & $\begin{array}{c}3,397 \\
(61.20)\end{array}$ & $0.0006^{*}$ & $569(60.28)$ & $\begin{array}{c}1,759 \\
(66.23)\end{array}$ & $<.0001 *$ \\
\hline Suburban & $\begin{array}{c}7,177 \\
(27.91)\end{array}$ & $\begin{array}{c}5,521 \\
(27.39)\end{array}$ & $\begin{array}{c}1,656 \\
(29.83)\end{array}$ & & $273(28.92)$ & $710(26.73)$ & \\
\hline Rural & $1,883(7.32)$ & $1,469(7.29)$ & $414(7.46)$ & & $70(7.42)$ & $164(6.17)$ & \\
\hline Unknown & $338(1.31)$ & $254(1.26)$ & $84(1.51)$ & & $32(3.39)$ & $23(0.87)$ & \\
\hline \multicolumn{8}{|l|}{ Income levels, No. (\%) } \\
\hline Quintile 1 & $\begin{array}{c}5,016 \\
(19.51)\end{array}$ & $\begin{array}{c}3,967 \\
(19.68)\end{array}$ & $\begin{array}{c}1,049 \\
(18.90)\end{array}$ & $<.0001 *$ & $233(24.68)$ & $383(14.42)$ & $<.0001 *$ \\
\hline Quintile 2 & $\begin{array}{c}5,583 \\
(21.71)\end{array}$ & $\begin{array}{c}4,651 \\
(23.07)\end{array}$ & $932(16.79)$ & & $346(36.65)$ & $240(9.04)$ & \\
\hline Quintile 3 & $\begin{array}{c}4,605 \\
(17.91)\end{array}$ & $\begin{array}{c}3,762 \\
(18.66)\end{array}$ & $843(15.19)$ & & $66(6.99)$ & $737(27.75)$ & \\
\hline Quintile 4 & $\begin{array}{c}5,033 \\
(19.58)\end{array}$ & $\begin{array}{c}4,028 \\
(19.98)\end{array}$ & $\begin{array}{c}1,005 \\
(18.10)\end{array}$ & & $143(15.15)$ & $592(22.29)$ & \\
\hline Quintile 5 & $\begin{array}{c}5,296 \\
(20.60)\end{array}$ & $\begin{array}{c}3,631 \\
(18.01)\end{array}$ & $\begin{array}{c}1,665 \\
(29.99)\end{array}$ & & $143(15.15)$ & $694(26.13)$ & \\
\hline Unknown & $178(0.69)$ & $121(0.60)$ & $57(1.03)$ & & $13(1.38)$ & $10(0.38)$ & \\
\hline \multicolumn{8}{|l|}{ Occupation, No. (\%) } \\
\hline $\begin{array}{l}\text { Dependents of the } \\
\text { insured individuals }\end{array}$ & $\begin{array}{c}5,879 \\
(22.87)\end{array}$ & $\begin{array}{c}4,967 \\
(24.64)\end{array}$ & $912(16.43)$ & $<.0001 *$ & $194(20.55)$ & $552(20.78)$ & $0.0047^{*}$ \\
\hline $\begin{array}{l}\text { Civil servants, } \\
\text { teachers, military } \\
\text { personnel and veterans }\end{array}$ & $1,258(4.89)$ & $859(4.26)$ & $399(7.19)$ & & $55(5.83)$ & $129(4.86)$ & \\
\hline $\begin{array}{l}\text { Non-manual } \\
\text { workers and } \\
\text { professionals }\end{array}$ & $\begin{array}{c}7,916 \\
(30.79)\end{array}$ & $\begin{array}{c}5,897 \\
(29.25)\end{array}$ & $\begin{array}{c}2,019 \\
(36.37)\end{array}$ & & $295(31.25)$ & $895(33.70)$ & \\
\hline Manual workers & $\begin{array}{c}8,629 \\
(33.56)\end{array}$ & $\begin{array}{c}7,001 \\
(34.73)\end{array}$ & $\begin{array}{c}1,628 \\
(29.33)\end{array}$ & & $347(36.76)$ & $852(32.08)$ & \\
\hline Other & $2,029(7.89)$ & $1,436(7.12)$ & $593(10.68)$ & & $53(5.61)$ & $228(8.58)$ & \\
\hline $\begin{array}{l}\text { Charlson index } \\
\text { (mean } \pm \text { standard } \\
\text { deviation) }\end{array}$ & $2.07 \pm 2.63$ & $1.90 \pm 2.51$ & $2.66 \pm 2.95$ & $<.0001 *$ & $1.68 \pm 2.32$ & $0.71 \pm 1.88$ & $<.0001^{*}$ \\
\hline
\end{tabular}

$* p<0.05$. 
Table 2: Crude and standardized prevalence (per 100,000 persons) of thyroid cancer from 1997 to 2012

\begin{tabular}{|c|c|c|c|c|c|c|c|c|c|}
\hline \multirow[t]{2}{*}{ Year } & \multicolumn{3}{|c|}{ Total } & \multicolumn{3}{|c|}{ Male } & \multicolumn{3}{|c|}{ Female } \\
\hline & $\mathbf{N}$ & Crude & Standardized & $\mathbf{N}$ & Crude & Standardized & $\mathbf{N}$ & Crude & Standardized \\
\hline 1997 & $21,084,565$ & $\begin{array}{c}21.1(20.46- \\
21.70)\end{array}$ & $\begin{array}{c}26.2(25.43- \\
27.03)\end{array}$ & $10,789,367$ & $\begin{array}{c}7.9(7.38 \\
-8.45)\end{array}$ & $\begin{array}{c}9.6(8.94- \\
10.29)\end{array}$ & $10,295,198$ & $\begin{array}{c}34.9(33.74- \\
36.02)\end{array}$ & $\begin{array}{c}42.4(40.98- \\
43.85)\end{array}$ \\
\hline 1998 & $21,284,097$ & $\begin{array}{c}25.1(24.43- \\
25.78)\end{array}$ & $\begin{array}{c}31.1(30.22- \\
31.94)\end{array}$ & $10,919,811$ & $\begin{array}{c}9.4(8.87 \\
-10.02)\end{array}$ & $\begin{array}{c}11.4(10.67- \\
12.13)\end{array}$ & $10,364,286$ & $\begin{array}{c}41.6(40.36- \\
42.85)\end{array}$ & $\begin{array}{c}50.2(48.69- \\
51.78)\end{array}$ \\
\hline 1999 & $21,376,883$ & $\begin{array}{c}29.3(28.55- \\
30.00)\end{array}$ & $\begin{array}{c}35.8(34.92- \\
36.76)\end{array}$ & $10,961,724$ & $\begin{array}{c}10.9 \\
(10.25- \\
11.48)\end{array}$ & $\begin{array}{c}13.0(12.19- \\
13.73)\end{array}$ & $10,415,159$ & $\begin{array}{c}48.7(47.32- \\
50.00)\end{array}$ & $\begin{array}{c}58.1(56.47- \\
59.76)\end{array}$ \\
\hline 2000 & $21,437,626$ & $\begin{array}{c}33.9(33.12- \\
34.68)\end{array}$ & $\begin{array}{c}41.1(40.16- \\
42.11)\end{array}$ & $10,964,786$ & $\begin{array}{c}12.2 \\
(11.58- \\
12.88)\end{array}$ & $\begin{array}{c}14.5(13.73- \\
15.35)\end{array}$ & $10,472,840$ & $\begin{array}{c}56.6(55.14- \\
58.03)\end{array}$ & $\begin{array}{c}67.0(65.27- \\
68.78)\end{array}$ \\
\hline 2001 & $21,549,709$ & $\begin{array}{c}38.3(37.49- \\
39.14)\end{array}$ & $\begin{array}{c}46.1(45.04- \\
47.09)\end{array}$ & $10,980,153$ & $\begin{array}{c}14.0 \\
(13.29- \\
14.69)\end{array}$ & $\begin{array}{c}16.4(15.56- \\
17.26)\end{array}$ & $10,569,556$ & $\begin{array}{c}63.6(62.07- \\
65.11)\end{array}$ & $\begin{array}{c}74.9(73.09- \\
76.77)\end{array}$ \\
\hline 2002 & $21,490,211$ & $\begin{array}{c}42.7(41.81- \\
43.55)\end{array}$ & $\begin{array}{c}50.8(49.77- \\
51.91)\end{array}$ & $10,911,148$ & $\begin{array}{c}15.7 \\
(14.93- \\
16.41)\end{array}$ & $\begin{array}{c}18.2(17.33- \\
19.11)\end{array}$ & $10,579,063$ & $\begin{array}{c}70.5(68.94- \\
72.14)\end{array}$ & $\begin{array}{c}82.6(80.66- \\
84.51)\end{array}$ \\
\hline 2003 & $21,530,783$ & $\begin{array}{c}47.2(46.30- \\
48.13)\end{array}$ & $\begin{array}{c}55.4(54.28- \\
56.49)\end{array}$ & $10,896,006$ & $\begin{array}{c}17.5 \\
(16.69- \\
18.26)\end{array}$ & $\begin{array}{c}20.0(19.08- \\
20.93)\end{array}$ & $10,634,777$ & $\begin{array}{c}77.7(76.01- \\
79.36)\end{array}$ & $\begin{array}{c}89.8(87.84- \\
91.81)\end{array}$ \\
\hline 2004 & $21,692,825$ & $\begin{array}{c}52.1(51.16- \\
53.08)\end{array}$ & $\begin{array}{c}60.0(58.86- \\
61.12)\end{array}$ & $10,942,756$ & $\begin{array}{c}19.4 \\
(18.52- \\
20.17)\end{array}$ & $\begin{array}{c}21.8(20.89- \\
22.79)\end{array}$ & $10,750,069$ & $\begin{array}{c}85.5(83.73- \\
87.23)\end{array}$ & $\begin{array}{c}97.1(95.11- \\
99.17)\end{array}$ \\
\hline 2005 & $21,787,801$ & $\begin{array}{c}57.1(56.04- \\
58.05)\end{array}$ & $\begin{array}{c}64.5(63.34- \\
65.65)\end{array}$ & $10,948,301$ & $\begin{array}{c}21.1 \\
(20.26- \\
21.98)\end{array}$ & $\begin{array}{c}23.5(22.55- \\
24.51)\end{array}$ & $10,839,500$ & $\begin{array}{c}93.3(91.52- \\
95.15)\end{array}$ & $\begin{array}{c}104.4(102.3- \\
106.5)\end{array}$ \\
\hline 2006 & $21,879,926$ & $\begin{array}{c}62.2(61.12- \\
63.21)\end{array}$ & $\begin{array}{c}69.1(67.93- \\
70.29)\end{array}$ & $10,958,204$ & $\begin{array}{c}23.2 \\
(22.26- \\
24.06)\end{array}$ & $\begin{array}{c}25.4(24.38- \\
26.39)\end{array}$ & $10,921,722$ & $\begin{array}{c}101.3(99.41- \\
103.2)\end{array}$ & $\begin{array}{c}111.7(109.6- \\
113.8)\end{array}$ \\
\hline 2007 & $21,947,660$ & $\begin{array}{c}68.2(67.12- \\
69.30)\end{array}$ & $\begin{array}{c}74.6(73.40- \\
75.82)\end{array}$ & $10,962,073$ & $\begin{array}{c}25.5 \\
(24.58- \\
26.47)\end{array}$ & $\begin{array}{c}27.5(26.49- \\
28.56)\end{array}$ & $10,985,587$ & $\begin{array}{c}110.8(108.8- \\
112.8)\end{array}$ & $\begin{array}{c}120.5(118.3- \\
122.6)\end{array}$ \\
\hline 2008 & $22,009,377$ & $\begin{array}{c}74.9(73.79- \\
76.08)\end{array}$ & $\begin{array}{c}80.6(79.38- \\
81.87)\end{array}$ & $10,962,089$ & $\begin{array}{c}28.2 \\
(27.20- \\
29.19)\end{array}$ & $\begin{array}{c}30.0(28.94- \\
31.08)\end{array}$ & $11,047,288$ & $\begin{array}{c}121.3(119.3- \\
123.4)\end{array}$ & $\begin{array}{c}129.9(127.7- \\
132.1)\end{array}$ \\
\hline 2009 & $21,994,317$ & $\begin{array}{c}82.8(81.64- \\
84.05)\end{array}$ & $\begin{array}{c}87.6(86.29- \\
88.85)\end{array}$ & $10,922,495$ & $\begin{array}{c}31.2 \\
(30.18- \\
32.28)\end{array}$ & $\begin{array}{c}32.7(31.59- \\
33.80)\end{array}$ & $11,071,822$ & $\begin{array}{c}133.8(131.6- \\
135.9)\end{array}$ & $\begin{array}{c}141.0(138.7- \\
143.3)\end{array}$ \\
\hline 2010 & $22,009,193$ & $\begin{array}{c}91.2(89.98- \\
92.50)\end{array}$ & $\begin{array}{c}94.5(93.22- \\
95.84)\end{array}$ & $10,900,482$ & $\begin{array}{c}34.9 \\
(33.78- \\
36.00)\end{array}$ & $\begin{array}{c}36.0(34.83- \\
37.12)\end{array}$ & $11,108,711$ & $\begin{array}{c}146.5(144.3- \\
148.8)\end{array}$ & $\begin{array}{c}151.5(149.2- \\
153.9)\end{array}$ \\
\hline 2011 & $21,979,916$ & $\begin{array}{c}99.8(98.43- \\
101.1)\end{array}$ & $\begin{array}{c}101.4(100.03- \\
102.72)\end{array}$ & $10,861,770$ & $\begin{array}{c}38.5 \\
(37.35- \\
39.69)\end{array}$ & $\begin{array}{c}39.1(37.88- \\
40.25)\end{array}$ & $11,118,146$ & $\begin{array}{c}159.6(157.2- \\
161.9)\end{array}$ & $\begin{array}{c}162.0(159.7- \\
164.4)\end{array}$ \\
\hline 2012 & $21,952,273$ & $\begin{array}{c}109.9 \\
(108.5- \\
111.3)\end{array}$ & $\begin{array}{c}109.9(108.49- \\
111.26)\end{array}$ & $10,828,933$ & $\begin{array}{c}43.0 \\
(41.80- \\
44.27)\end{array}$ & $\begin{array}{c}43.0(41.80- \\
44.27)\end{array}$ & $11,123,340$ & $\begin{array}{c}175.0(172.5- \\
177.4)\end{array}$ & $\begin{array}{c}175.0(172.5- \\
177.4)\end{array}$ \\
\hline
\end{tabular}

of thyroid cancer was 12.30 per 100,000 person-years in 2012, with an average APC of 5.1 (95\% confidence interval, 2.5 to 7.8 ) during 1997-2012, similar to the incidence and APC reported in the Surveillance, 
Table 3: Crude and standardized incidence (per 100,000 person-years) of thyroid cancer from 1997 to 2012

\begin{tabular}{|c|c|c|c|c|c|c|c|c|c|}
\hline \multirow[t]{2}{*}{ Year } & \multicolumn{3}{|c|}{ Total } & \multicolumn{3}{|c|}{ Male } & \multicolumn{3}{|c|}{ Female } \\
\hline & $\begin{array}{c}\text { Person- } \\
\text { years }\end{array}$ & Crude & Standardized & $\begin{array}{c}\text { Person- } \\
\text { years }\end{array}$ & Crude & Standardized & $\begin{array}{c}\text { Person- } \\
\text { years }\end{array}$ & Crude & Standardized \\
\hline 1997 & $20,243,975$ & $\begin{array}{c}4.66(4.37 \\
-4.96)\end{array}$ & $\begin{array}{l}5.66(5.29- \\
6.04)\end{array}$ & $10,270,668$ & $\begin{array}{c}1.63(1.38 \\
-1.87)\end{array}$ & $\begin{array}{c}1.93(1.63- \\
2.24)\end{array}$ & $9,973,307$ & $\begin{array}{l}7.79(7.24 \\
-8.34)\end{array}$ & $\begin{array}{c}9.30(8.62- \\
9.97)\end{array}$ \\
\hline 1998 & $20,691,870$ & $\begin{array}{l}5.60(5.28 \\
-5.92)\end{array}$ & $\begin{array}{c}6.76(6.36- \\
7.17)\end{array}$ & $10,572,162$ & $\begin{array}{l}2.28(1.99 \\
-2.57)\end{array}$ & $\begin{array}{c}2.62(2.28- \\
2.97)\end{array}$ & $10,119,708$ & $\begin{array}{l}9.07(8.48 \\
-9.66)\end{array}$ & $\begin{array}{c}10.79(10.07- \\
11.51)\end{array}$ \\
\hline 1999 & $20,835,124$ & $\begin{array}{l}5.88(5.55 \\
-6.21)\end{array}$ & $\begin{array}{c}7.09(6.68- \\
7.50)\end{array}$ & $10,666,163$ & $\begin{array}{c}2.17(1.89 \\
-2.45)\end{array}$ & $\begin{array}{c}2.64(2.28- \\
2.99)\end{array}$ & $10,168,961$ & $\begin{array}{c}9.78(9.18 \\
-10.39)\end{array}$ & $\begin{array}{c}11.42(10.69- \\
12.16)\end{array}$ \\
\hline 2000 & $20,855,149$ & $\begin{array}{l}6.08(5.75 \\
-6.41)\end{array}$ & $\begin{array}{c}7.20(6.79- \\
7.61)\end{array}$ & $10,667,910$ & $\begin{array}{c}2.48(2.19 \\
-2.78)\end{array}$ & $\begin{array}{c}2.94(2.57- \\
3.31)\end{array}$ & $10,187,239$ & $\begin{array}{c}9.85(9.24 \\
-10.45)\end{array}$ & $\begin{array}{c}11.35(10.63- \\
12.08)\end{array}$ \\
\hline 2001 & $20,855,851$ & $\begin{array}{l}6.11(5.78 \\
-6.45)\end{array}$ & $\begin{array}{c}7.23(6.82- \\
7.63)\end{array}$ & $10,651,291$ & $\begin{array}{l}2.52(2.21 \\
-2.82)\end{array}$ & $\begin{array}{l}2.92(2.55- \\
3.28)\end{array}$ & $10,204,560$ & $\begin{array}{c}9.87(9.26 \\
-10.48)\end{array}$ & $\begin{array}{c}11.42(10.70- \\
12.15)\end{array}$ \\
\hline 2002 & $20,905,475$ & $\begin{array}{l}6.32(5.98 \\
-6.66)\end{array}$ & $\begin{array}{c}7.35(6.94- \\
7.76)\end{array}$ & $10,647,131$ & $\begin{array}{l}2.84(2.52 \\
-3.16)\end{array}$ & $\begin{array}{c}3.27(2.89- \\
3.65)\end{array}$ & $10,258,343$ & $\begin{array}{c}9.94(9.33 \\
-10.55)\end{array}$ & $\begin{array}{c}11.33(10.62- \\
12.04)\end{array}$ \\
\hline 2003 & $21,081,693$ & $\begin{array}{l}5.90(5.57 \\
-6.23)\end{array}$ & $\begin{array}{c}6.69(6.31- \\
7.07)\end{array}$ & $10,695,909$ & $\begin{array}{c}2.47(2.17 \\
-2.77)\end{array}$ & $\begin{array}{l}2.80(2.45- \\
3.15)\end{array}$ & $10,385,784$ & $\begin{array}{c}9.44(8.85 \\
-10.03)\end{array}$ & $\begin{array}{c}10.49(9.81- \\
11.16)\end{array}$ \\
\hline 2004 & $21,267,429$ & $\begin{array}{l}6.38(6.04 \\
-6.72)\end{array}$ & $\begin{array}{c}7.12(6.73- \\
7.51)\end{array}$ & $10,752,053$ & $\begin{array}{c}2.73(2.42 \\
-3.05)\end{array}$ & $\begin{array}{c}3.02(2.67- \\
3.37)\end{array}$ & $10,515,375$ & $\begin{array}{c}10.10 \\
(9.49 \\
-10.71)\end{array}$ & $\begin{array}{c}11.12(10.44- \\
11.80)\end{array}$ \\
\hline 2005 & $21,401,764$ & $\begin{array}{l}6.49(6.15 \\
-6.83)\end{array}$ & $\begin{array}{c}7.21(6.82- \\
7.59)\end{array}$ & $10,782,313$ & $\begin{array}{c}2.60(2.29 \\
-2.90)\end{array}$ & $\begin{array}{l}2.86(2.51- \\
3.20)\end{array}$ & $10,619,451$ & $\begin{array}{c}10.44 \\
(9.83 \\
-11.06)\end{array}$ & $\begin{array}{c}11.45(10.76- \\
12.13)\end{array}$ \\
\hline 2006 & $21,500,636$ & $\begin{array}{c}7.05(6.69 \\
-7.40)\end{array}$ & $\begin{array}{c}7.75(7.35- \\
8.15)\end{array}$ & $10,790,603$ & $\begin{array}{c}3.23(2.89 \\
-3.56)\end{array}$ & $\begin{array}{c}3.51(3.14- \\
3.89)\end{array}$ & $10,710,033$ & $\begin{array}{c}10.90 \\
(10.27- \\
11.52)\end{array}$ & $\begin{array}{c}11.88(11.18- \\
12.57)\end{array}$ \\
\hline 2007 & $21,586,501$ & $\begin{array}{c}7.83(7.46 \\
-8.20)\end{array}$ & $\begin{array}{c}8.44(8.03- \\
8.85)\end{array}$ & $10,796,739$ & $\begin{array}{c}3.47(3.12 \\
-3.82)\end{array}$ & $\begin{array}{l}3.73(3.35- \\
\quad 4.12)\end{array}$ & $10,789,762$ & $\begin{array}{c}12.19 \\
(11.53- \\
12.85)\end{array}$ & $\begin{array}{c}13.03(12.31- \\
13.74)\end{array}$ \\
\hline 2008 & $21,649,667$ & $\begin{array}{c}8.81(8.42 \\
-9.21)\end{array}$ & $\begin{array}{c}9.38(8.96- \\
9.81)\end{array}$ & $10,797,325$ & $\begin{array}{c}3.60(3.24 \\
-3.96)\end{array}$ & $\begin{array}{c}3.80(3.42- \\
4.18)\end{array}$ & $10,852,342$ & $\begin{array}{c}14.00 \\
(13.29- \\
14.70)\end{array}$ & $\begin{array}{c}14.83(14.07- \\
15.58)\end{array}$ \\
\hline 2009 & $21,670,116$ & $\begin{array}{c}10.14 \\
(9.72 \\
-10.57)\end{array}$ & $\begin{array}{c}10.62(10.18- \\
11.07)\end{array}$ & $10,778,664$ & $\begin{array}{c}4.46(4.06 \\
-4.86)\end{array}$ & $\begin{array}{c}4.69(4.26- \\
5.11)\end{array}$ & $10,891,452$ & $\begin{array}{c}15.76 \\
(15.02- \\
16.51)\end{array}$ & $\begin{array}{c}16.41(15.63- \\
17.19)\end{array}$ \\
\hline 2010 & $21,693,590$ & $\begin{array}{c}10.28 \\
(9.85 \\
-10.71)\end{array}$ & $\begin{array}{c}10.58(10.13- \\
11.02)\end{array}$ & $10,757,963$ & $\begin{array}{l}4.81(4.39 \\
-5.22)\end{array}$ & $\begin{array}{l}4.96(4.53- \\
5.39)\end{array}$ & $10,935,627$ & $\begin{array}{c}15.66 \\
(14.92- \\
16.41)\end{array}$ & $\begin{array}{c}16.05(15.28- \\
16.81)\end{array}$ \\
\hline 2011 & $21,638,725$ & $\begin{array}{c}10.77 \\
(10.34- \\
11.21)\end{array}$ & $\begin{array}{c}10.92(10.47- \\
11.36)\end{array}$ & $10,700,141$ & $\begin{array}{c}4.95(4.53 \\
-5.37)\end{array}$ & $\begin{array}{c}5.03(4.60- \\
5.46)\end{array}$ & $10,938,584$ & $\begin{array}{c}16.46 \\
(15.70- \\
17.23)\end{array}$ & $\begin{array}{c}16.65(15.88- \\
17.42)\end{array}$ \\
\hline 2012 & $21,586,050$ & $\begin{array}{c}12.30 \\
(11.84- \\
12.77)\end{array}$ & $\begin{array}{c}12.30(11.84- \\
12.77)\end{array}$ & $10,651,206$ & $\begin{array}{c}5.62(5.17 \\
-6.07)\end{array}$ & $\begin{array}{l}5.62(5.17- \\
6.07)\end{array}$ & $10,934,844$ & $\begin{array}{c}18.81 \\
(18.00- \\
19.62)\end{array}$ & $\begin{array}{c}18.81(18.00- \\
19.62)\end{array}$ \\
\hline
\end{tabular}

Epidemiology, and End Results (SEER) database of the United States (age-standardized incidence rate, 13.98 per 100,000 person-years in 2012; APC, 5.5 during
1992-2012) [9]. Male patients presented at older age at diagnosis and increased more rapidly than female cases, with the female-to-male ratio of age-standardized 
Table 4: Joinpoint analysis of thyroid cancer prevalence (per 100,000 persons) by sex in Taiwan, 1997-2012

Thyroid cancer

Trend 1

Trend 2

Trend 3

prevalence

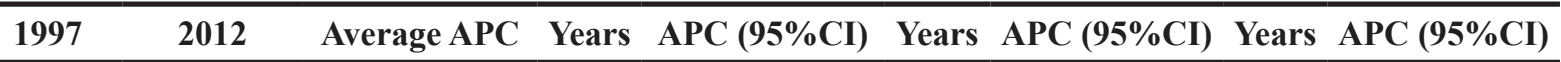

\section{Prevalence}

\begin{tabular}{lccccccccc} 
Total & $26.2(25.4$ & $109.9(108.5$ & $10.0(9.5$ to & $1997-$ & $17.5(14.7$ to & $1999-$ & $12.2(10.1$ to & 2002 \\
& to 27.0$)$ & to 111.3$)$ & $10.5)^{*}$ & 1999 & $20.3)^{*}$ & 2002 & $14.3)^{*}$ & -2012 & $7.9(7.8 \text { to } 8.0)^{*}$ \\
\multirow{2}{*}{ Male } & $9.6(8.9$ to & $43.0(41.8$ to & $9.9(9.4$ to & $1997-$ & $16.6(15.2$ to & $2000-$ & $10.0(7.8$ to & $2003-$ \\
& $10.3)$ & $44.3)$ & $10.3)^{*}$ & 2000 & $18.1)^{*}$ & 2003 & $12.1)^{*}$ & 2012 \\
\multirow{2}{*}{ Female } & $42.4(41.0$ & $175.0(172.5$ & $10.3(9.8$ to & $1997-$ & $14.1(12.3$ to & $2001-$ & & \\
& to 43.9$)$ & to 177.4$)$ & $10.7)^{*}$ & 2001 & $15.9)^{*}$ & 2012 & $8.9(8.7 \text { to } 9.1)^{*}$ & \\
\hline
\end{tabular}

APC, annual percent change; CI, confidence interval. $* P<0.05$.

(A) ALL

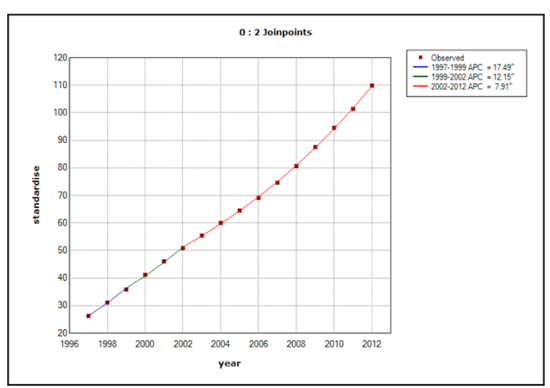

(B) Male

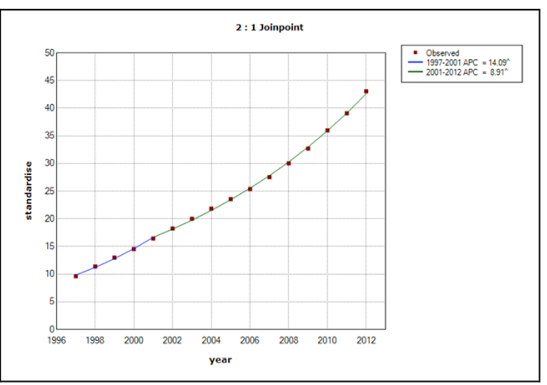

(C) Female

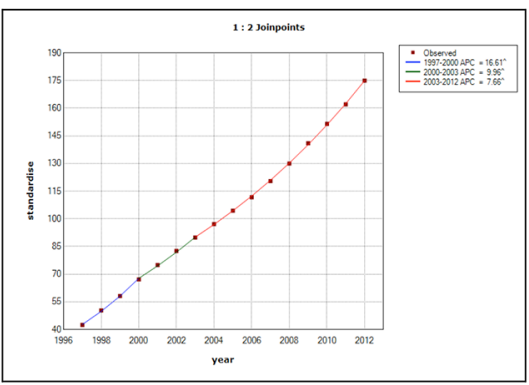

A

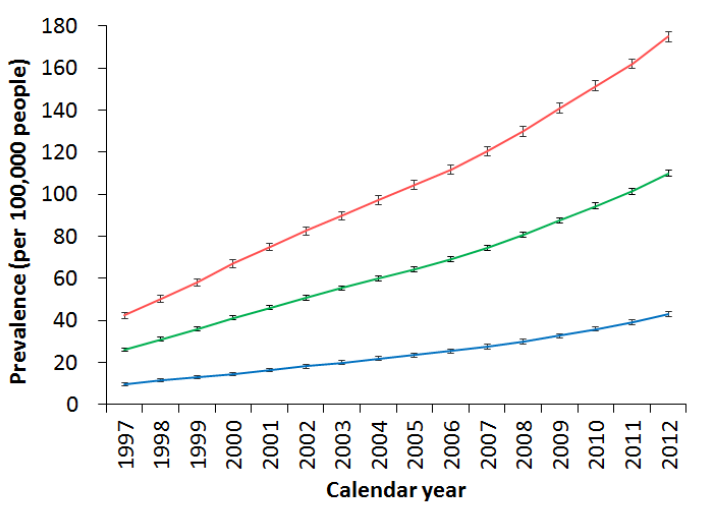

B

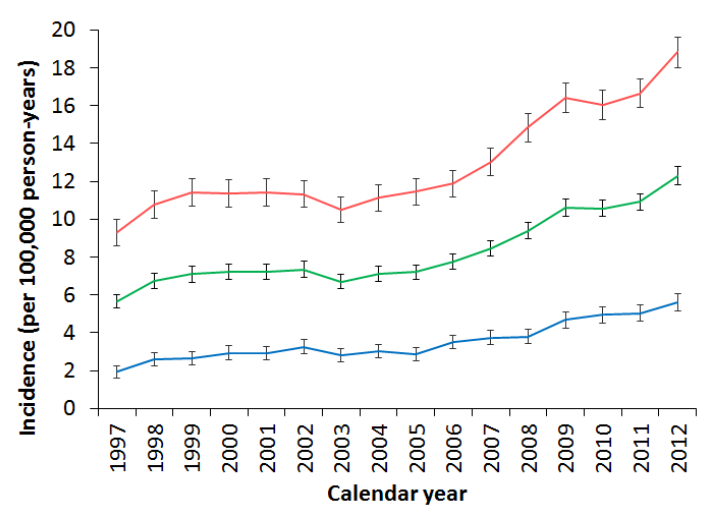

Figure 2: Differences by sex in the trends of (A) standardized prevalence and (B) standardized incidence of thyroid cancer in Taiwan between 1997 and 2012. (Red: female, green: total, blue: male.) 
Table 5: Joinpoint analysis of thyroid cancer incidence (per 100,000 person-years) by sex in Taiwan, $1997-2012$

\begin{tabular}{|c|c|c|c|c|c|c|c|c|c|}
\hline & \multicolumn{2}{|c|}{$\begin{array}{c}\text { Thyroid cancer } \\
\text { incidence }\end{array}$} & \multirow[b]{2}{*}{ Average APC } & \multicolumn{2}{|r|}{ Trend 1} & \multicolumn{2}{|r|}{ Trend 2} & \multicolumn{2}{|r|}{ Trend 3} \\
\hline & 1997 & 2012 & & Years & APC $(95 \%$ CI $)$ & Years & APC $(95 \%$ CI $)$ & Years & $\mathrm{APC}(95 \% \mathrm{CI})$ \\
\hline \multicolumn{10}{|c|}{ Incidence } \\
\hline Total & $\begin{array}{l}5.7(5.3 \\
\text { to } 6.0)\end{array}$ & $\begin{array}{c}12.3(11.8 \\
\text { to } 12.8)\end{array}$ & $5.1(2.5 \text { to } 7.8)^{*}$ & $\begin{array}{l}1997- \\
1999\end{array}$ & $\begin{array}{c}12.3(-5.9 \text { to } \\
34.0)\end{array}$ & $\begin{array}{l}1999- \\
2004\end{array}$ & $-1.1(-5.9$ to 4.1$)$ & $\begin{array}{l}2004- \\
2012\end{array}$ & $7.4(5.9 \text { to } 8.9)^{*}$ \\
\hline Male & $\begin{array}{l}1.9(1.6 \\
\text { to } 2.2)\end{array}$ & $\begin{array}{c}5.6(5.2 \text { to } \\
6.1)\end{array}$ & $\begin{array}{l}6.9(3.3 \text { to } \\
10.6)^{*}\end{array}$ & $\begin{array}{l}1997- \\
2000\end{array}$ & $\begin{array}{c}12.4(-2.7 \text { to } \\
29.8)\end{array}$ & $\begin{array}{l}2000- \\
2005\end{array}$ & $0.4(-6.9$ to 8.2$)$ & $\begin{array}{l}2005- \\
2012\end{array}$ & $\begin{array}{l}9.4(6.7 \text { to } \\
12.1)^{*}\end{array}$ \\
\hline Female & $\begin{array}{l}9.3(8.6 \\
\text { to } 10.0)\end{array}$ & $\begin{array}{c}18.8(18.0 \\
\text { to } 19.6)\end{array}$ & $4.6(1.8 \text { to } 7.5)^{*}$ & $\begin{array}{l}1997- \\
1999\end{array}$ & $\begin{array}{c}10.6(-8.5 \text { to } \\
33.7)\end{array}$ & $\begin{array}{l}1999- \\
2004\end{array}$ & $-1.3(-6.6$ to 4.2$)$ & $\begin{array}{l}2004- \\
2012\end{array}$ & $7.1(5.5 \text { to } 8.7)^{*}$ \\
\hline
\end{tabular}

APC, annual percent change; CI, confidence interval. $* P<0.05$.

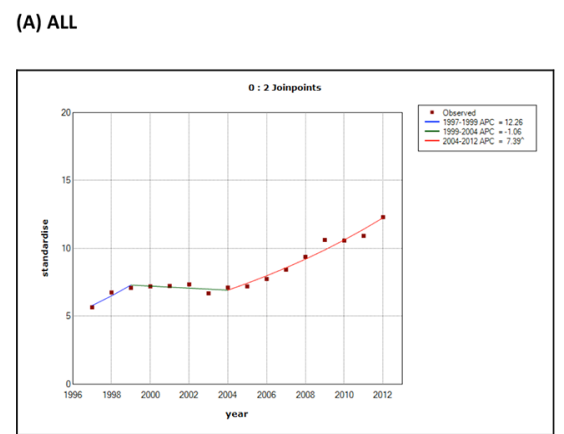

(B) Male

(C) Female
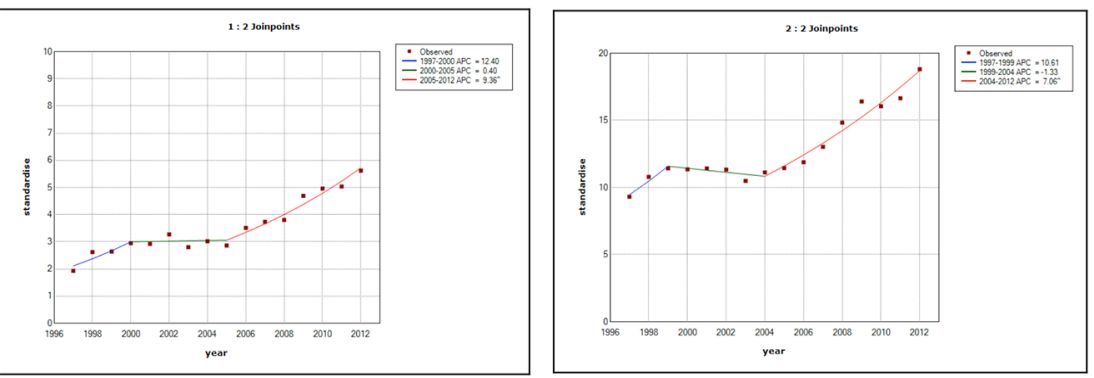

A

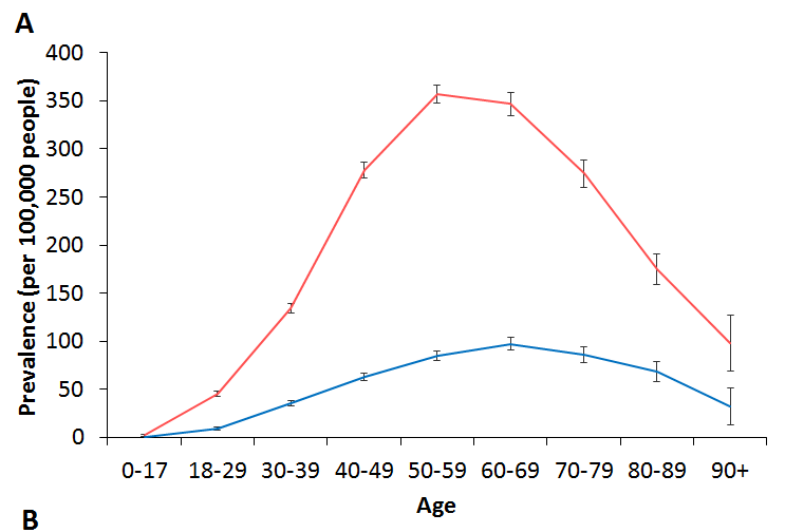

B

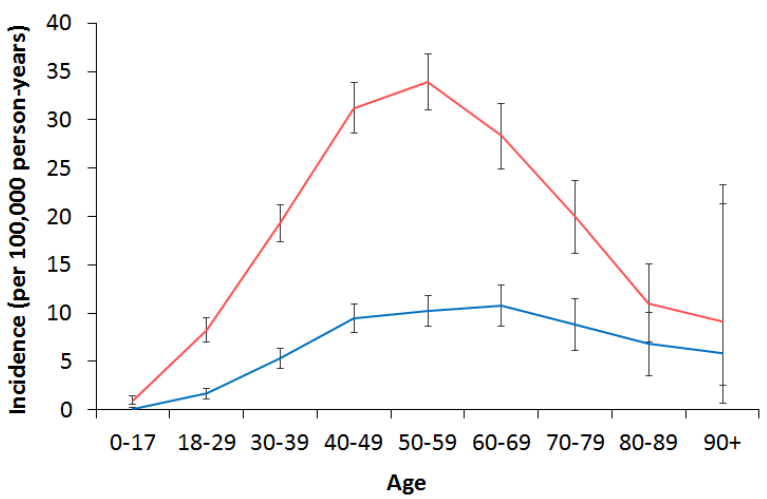

Figure 3: Differences by sex in the trends of (A) age-specific prevalence and (B) age-specific incidence of thyroid cancer in Taiwan between 1997 and 2012. (Red: female, blue: male.) 
Table 6: Demographic data of patients with different thyroid cancer subtypes in Taiwan from 1997 to 2012

\begin{tabular}{|c|c|c|c|c|c|}
\hline & $\begin{array}{l}\text { Papillary } \\
\text { thyroid } \\
\text { carcinoma }\end{array}$ & $\begin{array}{l}\text { Follicular } \\
\text { thyroid } \\
\text { carcinoma }\end{array}$ & $\begin{array}{l}\text { Medullary } \\
\text { thyroid } \\
\text { carcinoma }\end{array}$ & $\begin{array}{l}\text { Anaplastic } \\
\text { thyroid } \\
\text { carcinoma }\end{array}$ & $\begin{array}{c}\text { Other type } \\
\text { thyroid cancer }\end{array}$ \\
\hline $\begin{array}{l}\text { Age (years) (mean } \pm \\
\text { standard deviation) }\end{array}$ & $46.23 \pm 14.23$ & $48.87 \pm 18.22$ & $51.56 \pm 14.61$ & $70.58 \pm 11.74$ & $58.63 \pm 19.62$ \\
\hline \multicolumn{6}{|l|}{ Sex, No. (\%) } \\
\hline Female & $18,374(79.11)$ & $1,615(71.59)$ & $233(60.99)$ & $218(63.01)$ & $806(60.47)$ \\
\hline Male & $4,853(20.89)$ & $641(28.41)$ & $149(39.01)$ & $128(36.99)$ & $527(39.53)$ \\
\hline \multicolumn{6}{|l|}{ Place of residence, No. (\%) } \\
\hline Urban & $13,894(59.82)$ & $1,254(55.59)$ & $215(56.28)$ & $144(41.62)$ & $648(48.61)$ \\
\hline Suburban & $6,218(26.77)$ & $685(30.36)$ & $111(29.06)$ & $90(26.01)$ & $382(28.66)$ \\
\hline Rural & $1,542(6.64)$ & $189(8.38)$ & $34(8.90)$ & $37(10.69)$ & $165(12.38)$ \\
\hline Unknown & $1,573(6.77)$ & $128(5.67)$ & $22(5.76)$ & $75(21.68)$ & $138(10.35)$ \\
\hline \multicolumn{6}{|l|}{ Income levels, No. (\%) } \\
\hline Quintile 1 & $4,689(20.19)$ & $476(21.10)$ & $83(21.73)$ & $75(21.68)$ & $334(25.06)$ \\
\hline Quintile 2 & $3,990(17.18)$ & $488(21.63)$ & $75(19.63)$ & $74(21.39)$ & $352(26.41)$ \\
\hline Quintile 3 & $4,794(20.64)$ & $448(19.86)$ & $65(17.02)$ & $59(17.05)$ & $189(14.18)$ \\
\hline Quintile 4 & $4,601(19.81)$ & $410(18.17)$ & $82(21.47)$ & $37(10.69)$ & $169(12.68)$ \\
\hline Quintile 5 & $5,059(21.78)$ & $398(17.64)$ & $72(18.85)$ & $45(13.01)$ & $186(13.95)$ \\
\hline Unknown & $94(0.40)$ & $36(1.60)$ & $5(1.31)$ & $56(16.18)$ & $103(7.73)$ \\
\hline \multicolumn{6}{|l|}{ Occupation, No. (\%) } \\
\hline $\begin{array}{l}\text { Dependents of the insured } \\
\text { individuals }\end{array}$ & $5,100(21.96)$ & $661(29.3)$ & $81(21.2)$ & $117(33.82)$ & $392(29.41)$ \\
\hline $\begin{array}{l}\text { Civil servants, teachers, } \\
\text { military personnel and } \\
\text { veterans }\end{array}$ & $1,087(4.68)$ & $97(4.30)$ & $23(6.02)$ & $5(1.45)$ & $27(2.03)$ \\
\hline $\begin{array}{l}\text { Non-manual workers and } \\
\text { professionals }\end{array}$ & $7,381(31.78)$ & $558(24.73)$ & $103(26.96)$ & $18(5.20)$ & $150(11.25)$ \\
\hline Manual workers & $7,423(31.96)$ & $685(30.36)$ & $122(31.94)$ & $109(31.50)$ & $482(36.16)$ \\
\hline Other & $2,236(9.63)$ & $255(11.3)$ & $53(13.87)$ & $97(28.03)$ & $282(21.16)$ \\
\hline $\begin{array}{l}\text { Charlson index (mean } \pm \\
\text { standard deviation) }\end{array}$ & $0.72 \pm 1.65$ & $0.84 \pm 1.88$ & $1.05 \pm 2.13$ & $2.07 \pm 2.76$ & $1.74 \pm 2.55$ \\
\hline
\end{tabular}

incidences decreasing from 4.8 in 1997 to 3.3 in 2012. When comparing the demographic data of patients with different histological subtypes, patients with papillary thyroid carcinoma presented at a younger age, had less comorbidities, and had a higher socioeconomic status. The incident percentage of the papillary subtype increased the most during the study period (from $80.6 \%$ of the total cases in 1998 to $89.8 \%$ in 2012), and the papillary-to-follicular incidence ratio increased from 8.14 in 1997 to 14.13 in 2012, a ratio even higher than that seen in the SEER database (9.88 during 2005-2009)
[10]. Thyroid cancer most frequently affected those with high socioeconomic status, such as those with higher income level, urban residence, and professional occupation, and this disparity got even more pronounced in recent years. Regarding the treatment procedures, the numbers of partial thyroidectomies increased the most (average APC, 17.3), which also implied that the numbers of incident cases with smallsized thyroid tumors increased the most during this period. The survival rates of thyroid cancer patients by calendar year, sex, subtype, and CCI were essentially 
stable; however, patients in the subgroups of male sex, $\mathrm{CCI} \geq 4$, and aggressive histological subtypes showed worse prognoses. These findings were consistent with epidemiological studies conducted in other countries $[11,12]$. Combining the following observations in our study: the increasing thyroid cancer incidences but stable survival, predominant increase in the papillary subtype, and marked increase in patients with high socioeconomic status, our results imply that overdetection of subclinical lesions could largely explain the epidemic of thyroid cancer in Taiwan.

\section{Analysis of socioeconomic status}

Socioeconomic status is highly associated with individual's access to health care such as screening, preventive care, and treatments. If the observed increase in thyroid cancer incidences is due to enhanced detection, patients with high socioeconomic status would be expected to have a greater increase in the incidence of small papillary tumors, which has been observed in several cohort studies using the SEER database and coincident with our results [13-15]. However, for large

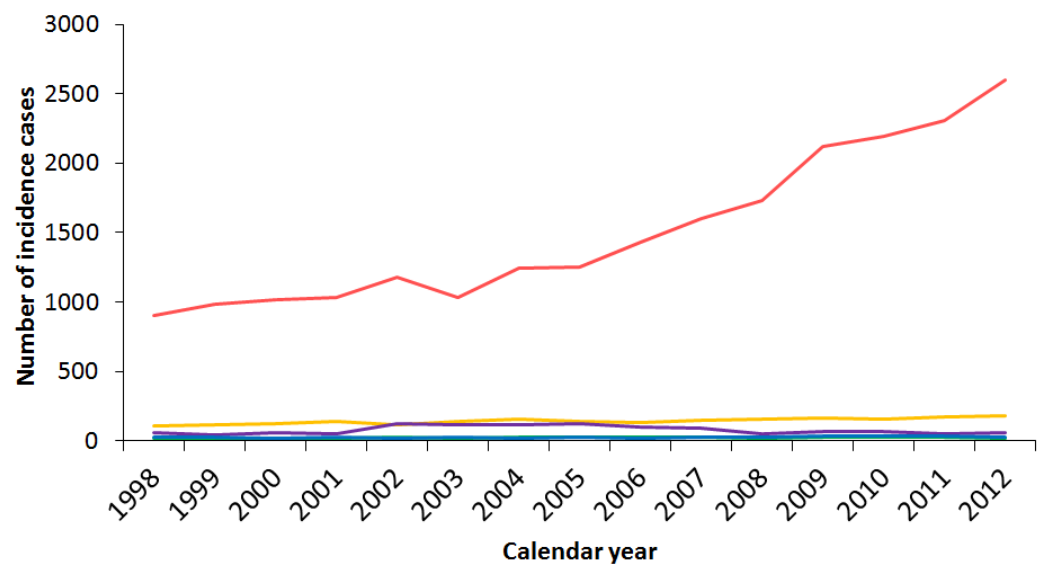

Figure 4: Secular trends of the incidence cases of different thyroid cancer subtypes in Taiwan between 1998 and 2012. (Red: papillary thyroid carcinoma, yellow: follicular thyroid carcinoma, blue: medullary thyroid carcinoma, green: anaplastic thyroid carcinoma, purple: other types.)

A

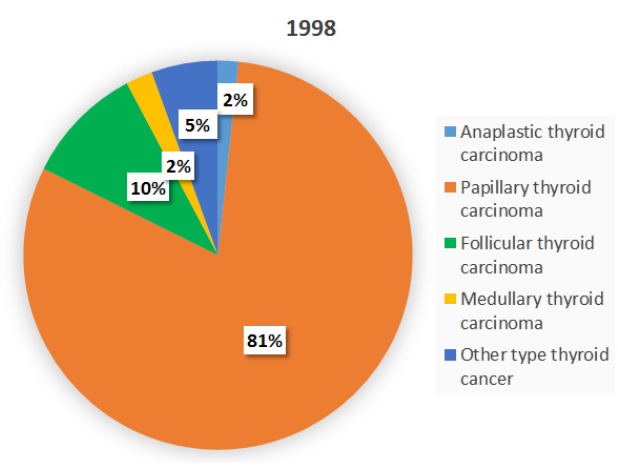

B

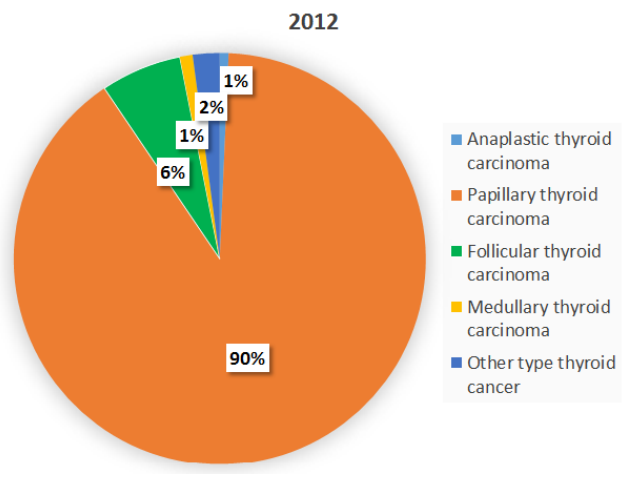

Figure 5: The percentages of different thyroid cancer subtypes in Taiwan in (A) 1998 and (B) 2012. 
Table 7: Joinpoint analysis of overall survival by different thyroid cancer subtypes in Taiwan, 1998-2012

\begin{tabular}{|c|c|c|c|}
\hline \multirow[t]{2}{*}{ Thyroid cancer subtypes } & \multicolumn{2}{|c|}{ Overall survival rate $(\%)$} & \multirow[t]{2}{*}{ Average APC } \\
\hline & 1998 & Last year $^{\mathrm{a}}$ & \\
\hline \multicolumn{4}{|l|}{ Papillary thyroid carcinoma } \\
\hline 1 year survival rate & $98.2 \%(96.92-98.91)$ & $98.7 \%(98.17-99.06)$ & $0.02(-0.01$ to 0.05$)$ \\
\hline 2 year survival rate & $97.2 \%(95.81-98.20)$ & $98.0 \%(97.42-98.50)$ & $0.03(-0.01$ to 0.07$)$ \\
\hline 5 year survival rate & $94.5 \%(92.62-95.90)$ & $95.5 \%(94.49-96.36)$ & $0.05(-0.04$ to 0.15$)$ \\
\hline \multicolumn{4}{|l|}{ Follicular thyroid carcinoma } \\
\hline 1 year survival rate & $95.3 \%(89.04-98.01)$ & $97.3 \%(93.56-98.85)$ & $0.05(-0.12$ to 0.22$)$ \\
\hline 2 year survival rate & $93.4 \%(86.65-96.80)$ & $95.1 \%(90.75-97.41)$ & $0.19(-0.07$ to 0.45$)$ \\
\hline 5 year survival rate & $86.8 \%(78.72-91.96)$ & $89.3 \%(81.36-93.99)$ & $0.24(-0.35$ to 0.84$)$ \\
\hline \multicolumn{4}{|l|}{ Medullary thyroid carcinoma } \\
\hline 1 year survival rate & $92.9 \%(59.08-98.96)$ & $89.7 \%(71.26-96.54)$ & $-0.30(-0.84$ to 0.23$)$ \\
\hline 2 year survival rate & $92.9 \%(59.08-98.96)$ & $89.7 \%(71.26-96.54)$ & $-0.72(-1.27 \text { to }-0.17)^{*}$ \\
\hline 5 year survival rate & $78.6 \%(47.25-92.54)$ & $80.6 \%(61.91-90.80)$ & $-0.20(-1.07$ to 0.68$)$ \\
\hline \multicolumn{4}{|l|}{ Anaplastic thyroid carcinoma } \\
\hline 1 year survival rate & $29.4 \%(10.71-51.15)$ & $13.8 \%(4.35-28.61)$ & $-5.51(-10.08 \text { to }-0.72)^{*}$ \\
\hline 2 year survival rate & $23.5 \%(7.31-44.92)$ & $6.90 \%(1.22-19.75)$ & $-7.45(-11.59 \text { to }-3.13)^{*}$ \\
\hline 5 year survival rate & $17.6 \%(4.35-38.30)$ & $7.40 \%(1.30-21.03)$ & $-17.34(-54.97$ to -0.68$)$ \\
\hline \multicolumn{4}{|l|}{ Other type thyroid cancer } \\
\hline 1 year survival rate & $71.2 \%(57.81-80.99)$ & $63.3 \%(49.85-74.11)$ & $-1.34(-3.15$ to 0.51$)$ \\
\hline 2 year survival rate & $69.5 \%(56.04-79.55)$ & $53.3 \%(40.01-64.96)$ & $-1.98(-4.28$ to 0.37$)$ \\
\hline 5 year survival rate & $64.4 \%(50.81-75.14)$ & $36.4 \%(23.84-49.00)$ & $-3.08(-6.53$ to 0.49$)$ \\
\hline
\end{tabular}

APC, annual percent change; $* P<0.05$.

${ }^{\text {a }}$ The last year for the 1-year and 2-year survival rate is 2012; the last year for the 5-year survival rate is 2010 .

tumors $(>4.0 \mathrm{~cm})$, a similar steady increase in incidence was also observed both for patients with high and low socioeconomic status in one previous study [13], indicating the existence of a true increase of thyroid cancer. In Canada, where a universal health care system is utilized, socioeconomic disparities of thyroid cancer were also reported $[16,17]$. Patients with thyroid cancer among those with low socioeconomic status were not only less frequently diagnosed but also presented with more advanced stage than patients with high socioeconomic status [17].

\section{Over-detection of subclinical thyroid cancer}

According to epidemiologic data from the global cancer registries of World Health Organization, there is an upward trend in the incidences of thyroid cancer (mainly papillary carcinomas) in both sexes and a stable or downward trend in mortality in most areas of the world [18]. The epidemic of thyroid cancer is often attributed to over-detection of small papillary lesions subsequent to improved diagnostic modalities, increased medical surveillances, and accessible "health checkup" services [19]. Generally, the increase of thyroid cancer incidences is faster in the developed high-income countries, with the exceptions of relatively modest increases in Denmark, the United Kingdom and Japan [12]. In the Asia-Pacific regions, the reported thyroid cancer incidences were higher in the high-income countries of Eastern and Western Asia with easier medical accessibilities, while the incidences were apparently low in the developing countries of South-Central Asia, such as India [2]. The epidemiological variations between different countries are usually ascribed to different health systems and screening practices [18]. Among the countries with reported epidemics of thyroid cancer, South Korea has by far the largest increase in the incidence of thyroid cancer. In South Korea, the incidence rate of thyroid cancer diagnosed in 2011 was 15 times higher compared to that observed in 1993, making thyroid cancer the most commonly diagnosed cancer among South Korean women 
Table 8: The numbers of different treatment procedures for patients with thyroid cancer by sex in Taiwan from 1997 to 2011

\begin{tabular}{lcccccc}
\hline Treatments $^{\mathbf{a}}$ & $\begin{array}{c}\text { Entire cohort } \\
(\mathbf{n}=\mathbf{2 5 7 1 1})\end{array}$ & \multicolumn{5}{c}{ By sex } \\
\cline { 3 - 7 } & & Female(n=20160) & \% in female pts & Male(n=5551) & \% in male pts & $\boldsymbol{p}$-Value \\
\hline Partial thyroidectomy & 8,573 & 6,832 & $33.89 \%$ & 1,741 & $31.36 \%$ & $0.0004^{*}$ \\
Total thyroidectomy & 13,649 & 10,708 & $53.12 \%$ & 2,941 & $52.98 \%$ & 0.8598 \\
Lymph node dissection & 6,972 & 5,237 & $25.98 \%$ & 1,735 & $31.26 \%$ & $<0.0001^{*}$ \\
Chemotherapy & 1,030 & 676 & $3.35 \%$ & 354 & $6.37 \%$ & $<0.0001^{*}$ \\
External beam & 2,600 & 1,906 & $9.45 \%$ & 694 & $12.5 \%$ & $<0.0001 *$ \\
radiotherapy & 15,718 & 12,305 & $61.04 \%$ & 3,413 & $61.48 \%$ & 0.5445 \\
\hline I-131 ablation & &
\end{tabular}

${ }^{a}$ All treatments for thyroid cancer occurred after the diagnosis of thyroid cancer. ${ }^{*} \mathrm{P}<0.05$.

$[20,21]$. These increasing diagnoses largely resulted from fee-for-service providers adding thyroid ultrasonography within the frame-work of organized screening programs for other cancers paid for by the South Korea government since 1999 [20]. Since then, there was 6.4-fold increase in thyroid cancer incidence in South Korea between 1999 and 2008 , of which $94.4 \%$ were small tumors $(<20 \mathrm{~mm}$ ) mainly detected by screening, and $97.1 \%$ of the total increase were locoregional tumors, with five-year relative survival rates over $100 \%$ [22].

Using tumor size at diagnosis, the estimated proportion of overdiagnosis in women during 20032007 may account for $90 \%$ of thyroid cancer incidences in South Korea; more than $70 \%$ in the United States, France, Italy, and Australia; and for 50\% in Japan [21, 23]. The proportions attributable to increased detection are higher in countries with larger incidence increases and are consistent across both sexes, although the reported increases were often smaller and delayed in men. In another study, the cancer stage at diagnosis was used to evaluate the overdiagnosis of papillary thyroid carcinoma in the SEER database [6]; as a result, the reported proportions of overdiagnosis in 2011 were $5.5 \%$ and $45.5 \%$ in men aged $20-49$ and $>50$ years, and $41.1 \%$ and $60.1 \%$ in women aged 20-49 and $>50$ years, respectively. Overdiagnosis, established by the surplus of early stage diseases outpacing late stage diseases, has resulted in an additional 82,000 incident papillary thyroid cancers during 1981-2011 in the US, and the majority of over-diagnosed patients will not benefit from surgical or adjuvant interventions due to their inherently favorable prognosis [6]. In our study, estimating the magnitude of overdiagnosis was impossible, since the databases used contained no information on the tumor size or cancer stage at diagnosis.

\section{True thyroid cancer increase: possible causative risk factors}

The proposed risk factors of thyroid cancer in the literatures include females, family history of thyroid cancer, radiation exposure, excess weight, iodine insufficiency and dietary factors [12]. However, available evidences on the risk factors of thyroid cancer were limited and heterogeneous. Tobacco use and alcohol consumption were associated with a slightly reduced risk of thyroid cancer in several cohort studies [24, 25]. Diabetes and excess weight have been recognized as risk factors for increased the incidence and mortality of thyroid cancer [26]. In a pooled analysis of 22 prospective cohort studies, greater body height and excess weight throughout adulthood were found to be associated with higher incidences of thyroid cancer (except the medullary subtype), and higher thyroid cancer mortality [27]. Since the incidence of most malignancies did not increase during the same period, the true carcinogens behind the epidemics of thyroid cancer must be thyroid-specific rather than common causes of other cancers such as diabetes, obesity, or viruses. A true increase of thyroid cancer incidence in recent decades, driven by the emergence of potential thyroid-specific risk factors such as radiation exposure, iodine insufficiency, or environmental carcinogens, is supported by molecular and epidemiological evidences.

The molecular evidence comes from a histological study analyzing genetic variations of papillary thyroid carcinomas in a United States institution from 1974 to 2009 , which showed sharply rising percentages of the follicular variant histology (from $18 \%$ to $44 \%$ ) and RAS mutations (from $3 \%$ to $25 \%$ ), overall stable prevalence of BRAF mutations but increased percentage within the classic papillary type (from $50 \%$ to $77 \%$ ), and a decreased proportion of RET/PTC rearrangements (11\% to $2 \%$ ) [28]. These results not only suggest the existence of new and more recent etiologic factors resulting in the change of molecular profiling and the increased incidence of thyroid cancer, but also imply that environmental or therapeutic radiation is not likely to be the major contributor, since as many as $80 \%$ of papillary thyroid carcinoma patients with radiation exposure carry RET/PTC chromosomal rearrangements $[3,29]$. 
Table 9: Joinpoint analysis of the number of treatment procedures for thyroid cancer in Taiwan, 1998-2011

\begin{tabular}{|c|c|c|c|c|c|c|c|c|c|}
\hline & \multicolumn{3}{|c|}{$\begin{array}{l}\text { Number of } \\
\text { treatments }\end{array}$} & \multicolumn{2}{|r|}{ Trend 1} & \multicolumn{2}{|r|}{ Trend 2} & \multicolumn{2}{|c|}{ Trend 3} \\
\hline & 1998 & 2011 & Average APC & Years & APC $(95 \% C I)$ & Years & APC $(95 \% C I)$ & Years & $\begin{array}{c}\text { APC } \\
(95 \% \text { CI })\end{array}$ \\
\hline $\begin{array}{l}\text { Partial } \\
\text { thyroidectomy }\end{array}$ & 65 & 749 & $\begin{array}{c}17.3(14.2 \text { to } \\
20.5)^{*}\end{array}$ & $\begin{array}{l}1998- \\
2000\end{array}$ & $\begin{array}{c}119.3(81.5 \text { to } \\
165.1)^{*}\end{array}$ & $\begin{array}{l}2000- \\
2011\end{array}$ & $\begin{array}{l}4.71(3.38 \text { to } \\
6.06)^{*}\end{array}$ & & \\
\hline $\begin{array}{l}\text { Total } \\
\text { thyroidectomy }\end{array}$ & 502 & 1353 & $\begin{array}{c}8.39(5.93 \text { to } \\
10.9)^{*}\end{array}$ & $\begin{array}{l}1998- \\
2000\end{array}$ & $\begin{array}{c}15.0(-0.05 \text { to } \\
32.3)\end{array}$ & $\begin{array}{l}2000- \\
2006\end{array}$ & $\begin{array}{c}2.64(-0.53 \text { to } \\
5.91)\end{array}$ & $\begin{array}{l}2006- \\
2011\end{array}$ & $\begin{array}{l}13.0(9.51 \\
\text { to } 16.6)^{*}\end{array}$ \\
\hline $\begin{array}{l}\text { Lymph node } \\
\text { dissection }\end{array}$ & 194 & 788 & $\begin{array}{c}9.75(8.07 \text { to } \\
11.5)^{*}\end{array}$ & $\begin{array}{l}1998- \\
2011\end{array}$ & $\begin{array}{c}9.75(8.07 \text { to } \\
11.5)^{*}\end{array}$ & & & & \\
\hline Chemotherapy & 12 & 100 & $\begin{array}{c}13.6(9.51 \text { to } \\
17.9)^{*}\end{array}$ & $\begin{array}{l}1998- \\
2011\end{array}$ & $\begin{array}{c}13.6(9.51 \text { to } \\
17.9)^{*}\end{array}$ & & & & \\
\hline $\begin{array}{l}\text { External beam } \\
\text { radiotherapy }\end{array}$ & 161 & 165 & $\begin{array}{c}-2.54(-6.91 \text { to } \\
2.04)\end{array}$ & $\begin{array}{l}1998- \\
2011\end{array}$ & $\begin{array}{c}-2.54(-6.91 \text { to } \\
2.04)\end{array}$ & & & & \\
\hline I-131 ablation & 670 & 1524 & $\begin{array}{c}6.32(4.09 \text { to } \\
8.59)^{*}\end{array}$ & $\begin{array}{l}1998- \\
2004\end{array}$ & $\begin{array}{c}2.99(-0.94 \text { to } \\
7.07)\end{array}$ & $\begin{array}{l}2004- \\
2011\end{array}$ & $\begin{array}{c}9.26(5.95 \text { to } \\
12.7)^{*}\end{array}$ & & \\
\hline
\end{tabular}

APC, annual percent change; CI, confidence interval. $* \mathrm{P}<0.05$.
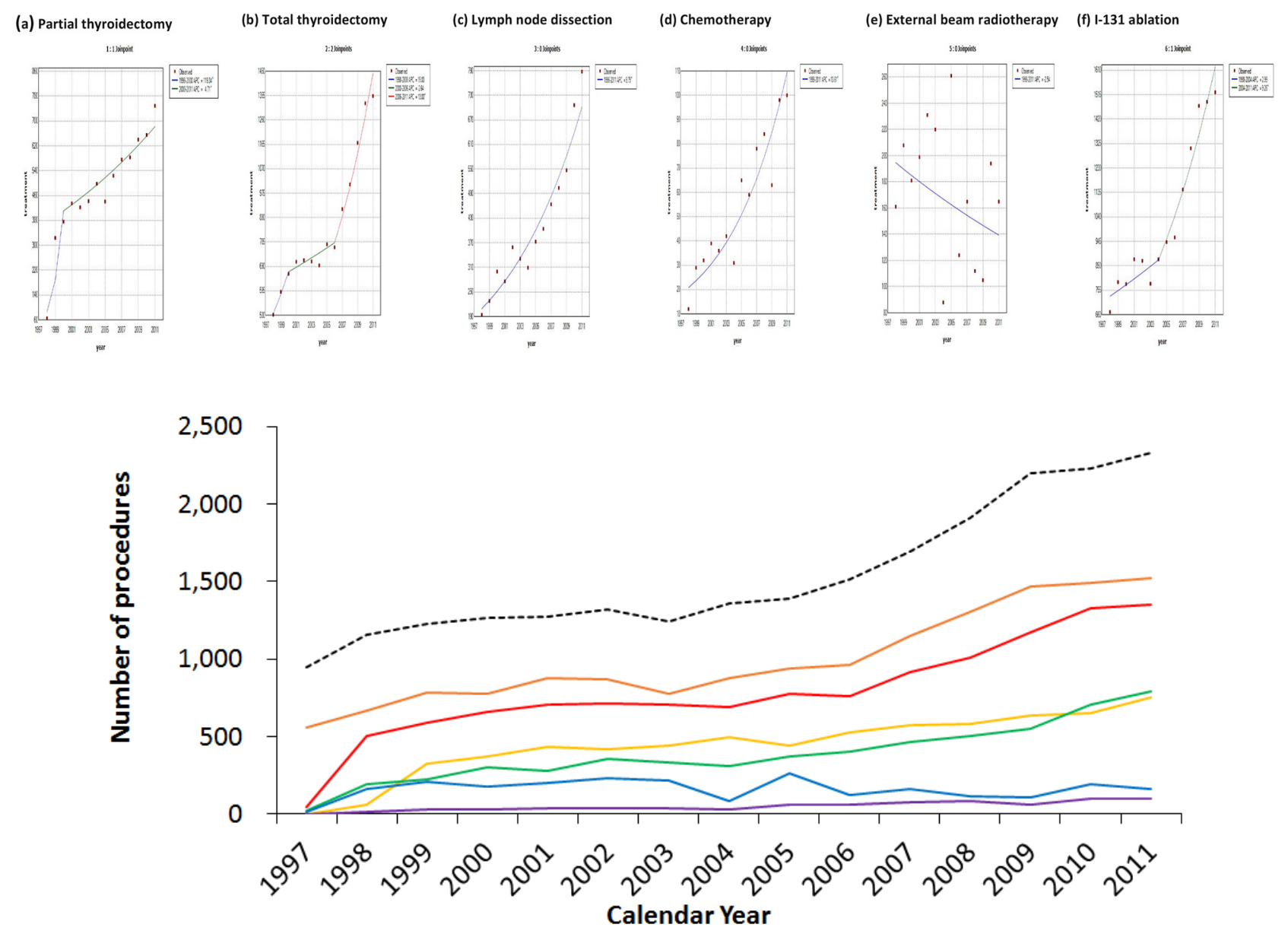

Figure 6: Secular trends of the numbers of different treatment procedures for thyroid cancer in Taiwan between 1997 and 2011. (Dotted line: the incidence cases at that specific year, orange: I-131 ablation, red: total thyroidectomy, yellow: partial thyroidectomy, green: lymph node dissection, blue: external beam radiotherapy, purple: chemotherapy.) 
On the other hand, epidemiological observations suggesting a true increase of thyroid cancer incidences include the following: flat mortality rates of thyroid cancer despite earlier diagnosis and more effective therapies [1], similar steady increase in incidence of large thyroid tumors $(>4.0 \mathrm{~cm}$ ) both in patients with high and low socioeconomic status [14], and the sharp increase in the incidence of papillary thyroid carcinomas 4-5 years after the nuclear-reactor accident in Chernobyl in 1986 [30].

Among these risks factors of thyroid cancer, exposure to ionizing radiation appears to be the most important contributor for papillary thyroid carcinoma with strong evidences support. The thyroid gland is highly sensitive to the carcinogenic effects of nuclear radiation, including diagnostic radiography, nuclear medicine, radiation therapy, and cosmic radiation during flights. After the Fukushima power plant accident in 2011, intensive ultrasonography screening programs within 4 years revealed that the thyroidcancer incidence among screened adolescents ( $\leq 18$ years) was approximately 30 times as high as the national average [31]. The nuclear radiation exposure dosage and age at exposure are key factors for the risk of radiation-induced thyroid cancer, and continuous monitoring accompanied with strict regulations of radiation exposure should be emphasized to prevent any health hazards. In Taiwan, a recent study comparing the incidence rates of leukemia and thyroid, lung, and breast cancers between patients in the "plant-vicinity" and "non-plant-vicinity" found no significant differences [32]. These results are consistent with our finding that there was no significant association between the geographic variation of thyroid cancer prevalence and the sites of nuclear power plants in Taiwan. For most people living far from the nuclear reactor/disasters, medical radiations make up a significant proportion of exposure, and its association with thyroid cancer has been exemplified in several studies that healthcare professionals had increased risks of thyroid cancer [33]. Besides, the use of radioactive iodine was associated with higher risk of secondary malignancy among thyroid cancer survivors [34].

Chronic iodine deficiency is firmly established as a risk factor for goiter and follicular thyroid carcinoma [3]. There might be a shift from the follicular to the papillary subtype after decades of universal prophylactic iodization programs [3]. In Taiwan, iodine sufficiency has been maintained since the mandatory salt iodization program implemented in 1967 and provides an essential element of thyroid hormones [8]. However, the iodization policy changed from mandatory to voluntary since 2003 in Taiwan, and the iodine status of Taiwanese populations has become mild insufficiency according to an adult urine iodine survey during 2005-2008 in all geographic areas (except the Southern area and Penghu islands), with significant insufficiency in subgroups of older age and mountain areas [35]. However, iodine deficiency

A

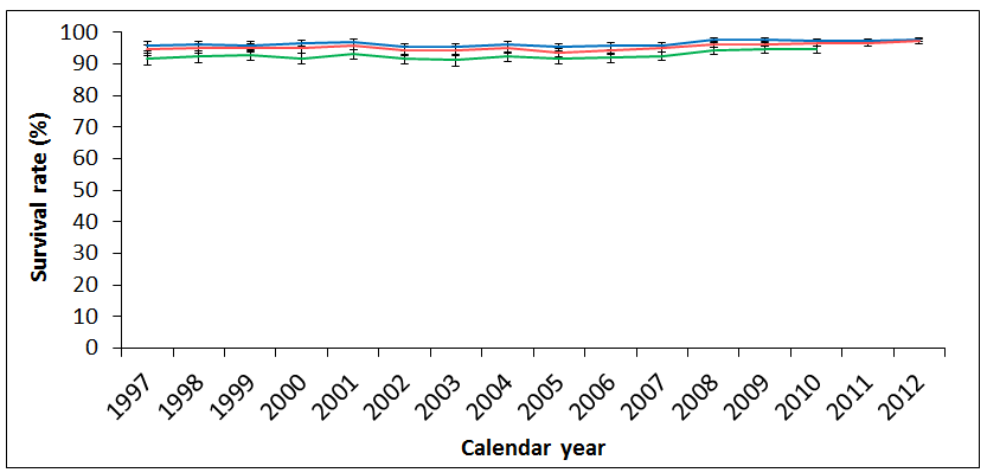

B

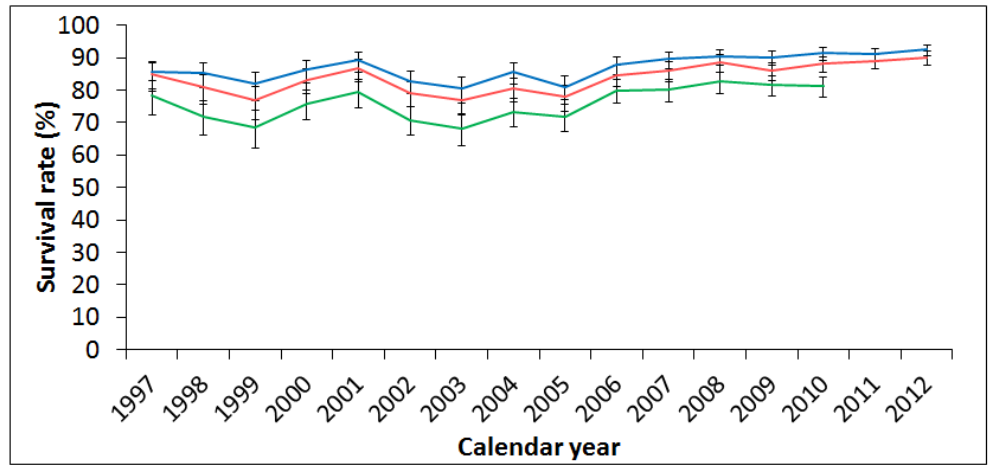

Figure 7: Secular trends of the one-, two-, and five-year survival rates of thyroid cancer in (A) female and (B) male patients in Taiwan from 1997 to 2012. (Blue: 1-year survival rate, red: 2-year survival rate, green: 5-year survival rate.) 
Table 10: Joinpoint analyses of thyroid cancer survival by calendar year, sex, and Charlson Comorbidity Index (CCI) in Taiwan, 1997-2012

\begin{tabular}{|c|c|c|c|c|c|c|c|c|c|c|}
\hline & & \multicolumn{2}{|c|}{$\begin{array}{c}\text { Thyroid cancer overall } \\
\text { survival }\end{array}$} & \multirow[t]{2}{*}{$\begin{array}{c}\text { Average } \\
\text { APC }\end{array}$} & \multicolumn{2}{|c|}{ Trend1 } & \multicolumn{2}{|c|}{ Trend2 } & \multicolumn{2}{|c|}{ Trend3 } \\
\hline & & 1997 & Last year ${ }^{\mathrm{a}}$ & & Years & $\begin{array}{c}\text { APC } \\
(95 \% C I)\end{array}$ & Years & $\begin{array}{c}\text { APC } \\
(95 \% C I)\end{array}$ & Years & $\begin{array}{c}\text { APC } \\
(95 \% \text { CI })\end{array}$ \\
\hline \multirow[t]{3}{*}{ Total } & $\begin{array}{l}1 \text { year } \\
\text { survival rate }\end{array}$ & $\begin{array}{c}94.9 \%(93.30 \\
\quad \text { to } 96.11)\end{array}$ & $\begin{array}{c}97.1 \%(96.44 \\
\quad \text { to } 97.67)\end{array}$ & $\begin{array}{l}0.19(0.08 \\
\text { to } 0.31)^{*}\end{array}$ & $\begin{array}{l}1997- \\
2012\end{array}$ & $\begin{array}{c}0.19(0.08 \\
\text { to } 0.31)^{*}\end{array}$ & & & & \\
\hline & $\begin{array}{l}2 \text { year } \\
\text { survival rate }\end{array}$ & $\begin{array}{c}93.7 \%(92.02 \\
\text { to } 95.11)\end{array}$ & $\begin{array}{c}96.1 \%(95.38 \\
\text { to } 96.79)\end{array}$ & $\begin{array}{l}0.16(-0.09 \\
\quad \text { to } 0.42)\end{array}$ & $\begin{array}{l}1997- \\
2004\end{array}$ & $\begin{array}{c}-0.25(-0.77 \\
\text { to } 0.28)\end{array}$ & $\begin{array}{l}2004- \\
2012\end{array}$ & $\begin{array}{c}0.53(0.24 \\
\text { to } 0.81)^{*}\end{array}$ & & \\
\hline & $\begin{array}{l}5 \text { year } \\
\text { survival rate }\end{array}$ & $\begin{array}{c}90.2 \%(88.14 \\
\text { to } 91.92)\end{array}$ & $\begin{array}{c}92.4 \%(91.22 \\
\text { to } 93.47)\end{array}$ & $\begin{array}{c}0.20(-0.18 \\
\text { to } 0.57)\end{array}$ & $\begin{array}{l}1997- \\
2003\end{array}$ & $\begin{array}{c}-0.45(-1.21 \\
\text { to } 0.32)\end{array}$ & $\begin{array}{l}2003- \\
2010\end{array}$ & $\begin{array}{c}0.75(0.30 \\
\text { to } 1.22)^{*}\end{array}$ & & \\
\hline \multirow[t]{8}{*}{ Sex } & Male & & & & & & & & & \\
\hline & $\begin{array}{l}1 \text { year } \\
\text { survival rate }\end{array}$ & $\begin{array}{l}90.7 \%(85.61 \\
\quad \text { to } 94.02)\end{array}$ & $\begin{array}{c}94.5 \%(92.46 \\
\text { to } 96.00)\end{array}$ & $\begin{array}{c}0.44(0.17 \\
\text { to } 0.71)^{*}\end{array}$ & $\begin{array}{l}1997- \\
2012\end{array}$ & $\begin{array}{c}0.44(0.17 \\
\text { to } 0.71)^{*}\end{array}$ & & & & \\
\hline & $\begin{array}{l}2 \text { year } \\
\text { survival rate }\end{array}$ & $\begin{array}{c}90.2 \%(85.00 \\
\text { to } 93.60)\end{array}$ & $\begin{array}{c}92.4 \%(90.05 \\
\quad \text { to } 94.16)\end{array}$ & $\begin{array}{l}0.50(0.14 \\
\text { to } 0.86)^{*}\end{array}$ & $\begin{array}{l}1997- \\
2012\end{array}$ & $\begin{array}{c}0.50(0.14 \\
\text { to } 0.86)^{*}\end{array}$ & & & & \\
\hline & $\begin{array}{l}5 \text { year } \\
\text { survival rate }\end{array}$ & $\begin{array}{l}84.5 \%(78.53 \\
\quad \text { to } 88.86)\end{array}$ & $\begin{array}{c}84.9 \%(81.36 \\
\quad \text { to } 87.74)\end{array}$ & $\begin{array}{l}0.66(0.00 \\
\text { to } 1.32)^{*}\end{array}$ & $\begin{array}{l}1997- \\
2010\end{array}$ & $\begin{array}{l}0.66(0.00 \\
\text { to } 1.32)^{*}\end{array}$ & & & & \\
\hline & Female & & & & & & & & & \\
\hline & $\begin{array}{l}1 \text { year } \\
\text { survival rate }\end{array}$ & $\begin{array}{c}96 \%(94.29 \\
\text { to } 97.14)\end{array}$ & $\begin{array}{c}97.9 \%(97.20 \\
\quad \text { to } 98.41)\end{array}$ & $\begin{array}{l}0.14(0.06 \\
\text { to } 0.21)^{*}\end{array}$ & $\begin{array}{l}1997- \\
2012\end{array}$ & $\begin{array}{c}0.14(0.06 \\
\text { to } 0.21)^{*}\end{array}$ & & & & \\
\hline & $\begin{array}{l}2 \text { year } \\
\text { survival rate }\end{array}$ & $\begin{array}{c}94.6 \%(92.80 \\
\quad \text { to } 96.03)\end{array}$ & $\begin{array}{c}97.3 \%(96.49 \\
\text { to } 97.86)\end{array}$ & $\begin{array}{l}0.15(0.02 \\
\text { to } 0.28)^{*}\end{array}$ & $\begin{array}{l}1997- \\
2005\end{array}$ & $\begin{array}{c}-0.09(-0.31 \\
\text { to } 0.13)\end{array}$ & $\begin{array}{l}2005- \\
2012\end{array}$ & $\begin{array}{c}0.42(0.24 \\
\text { to } 0.60)^{*}\end{array}$ & & \\
\hline & $\begin{array}{l}5 \text { year } \\
\text { survival rate }\end{array}$ & $\begin{array}{c}91.6 \%(89.45 \\
\quad \text { to } 93.40)\end{array}$ & $\begin{array}{c}94.8 \%(93.61 \\
\text { to } 95.76)\end{array}$ & $\begin{array}{l}0.23(0.01 \\
\text { to } 0.46)^{*}\end{array}$ & $\begin{array}{l}1997- \\
2006\end{array}$ & $\begin{array}{c}-0.03(-0.29 \\
\text { to } 0.22)\end{array}$ & $\begin{array}{l}2006- \\
2010\end{array}$ & $\begin{array}{c}0.84(0.24 \\
\text { to } 1.45)^{*}\end{array}$ & & \\
\hline \multirow[t]{8}{*}{ CCI } & $\mathrm{CCI} \leq 3$ & & & & & & & & & \\
\hline & $\begin{array}{l}1 \text { year } \\
\text { survival rate }\end{array}$ & $\begin{array}{c}96.3 \%(94.90 \\
\text { to } 97.27)\end{array}$ & $\begin{array}{c}97.9 \%(97.30 \\
\text { to } 98.38)\end{array}$ & $\begin{array}{l}0.15(0.05 \\
\text { to } 0.25)^{*}\end{array}$ & $\begin{array}{l}1997- \\
2012\end{array}$ & $\begin{array}{l}0.15(0.05 \\
\text { to } 0.25)^{*}\end{array}$ & & & & \\
\hline & $\begin{array}{l}2 \text { year } \\
\text { survival rate }\end{array}$ & $\begin{array}{c}94.6 \%(93.01 \\
\text { to } 95.82)\end{array}$ & $\begin{array}{c}97.2 \%(96.48 \\
\quad \text { to } 97.73)\end{array}$ & $\begin{array}{l}0.26(-0.25 \\
\text { to } 0.77)\end{array}$ & $\begin{array}{l}1997- \\
2000\end{array}$ & $\begin{array}{c}0.86(-0.59 \\
\text { to } 2.34)\end{array}$ & $\begin{array}{l}2000- \\
2003\end{array}$ & $\begin{array}{c}-0.94 \\
(-3.45 \text { to } \\
1.64)\end{array}$ & $\begin{array}{l}2003- \\
2012\end{array}$ & $\begin{array}{l}0.46(0.29 \\
\text { to } 0.63)^{*}\end{array}$ \\
\hline & $\begin{array}{l}5 \text { year } \\
\text { survival rate }\end{array}$ & $\begin{array}{l}91.4 \%(89.55 \\
\quad \text { to } 93.01)\end{array}$ & $\begin{array}{c}94.3 \%(93.18 \\
\text { to } 95.20)\end{array}$ & $\begin{array}{c}0.41(-0.37 \\
\text { to } 1.18)\end{array}$ & $\begin{array}{l}1997- \\
2000\end{array}$ & $\begin{array}{c}1.16(-0.84 \\
\text { to } 3.20)\end{array}$ & $\begin{array}{l}2000- \\
2003\end{array}$ & $\begin{array}{c}-1.28 \\
(-4.74 \text { to } \\
2.31)\end{array}$ & $\begin{array}{l}2003- \\
2010\end{array}$ & $\begin{array}{c}0.81(0.44 \\
\text { to } 1.19)^{*}\end{array}$ \\
\hline & $\mathrm{CCI} \geq 4$ & & & & & & & & & \\
\hline & $\begin{array}{l}1 \text { year } \\
\text { survival rate }\end{array}$ & $\begin{array}{l}81.4 \%(72.18 \\
\quad \text { to } 87.88)\end{array}$ & $\begin{array}{c}83.6 \%(76.92 \\
\quad \text { to } 88.56)\end{array}$ & $\begin{array}{c}0.20(-0.33 \\
\text { to } 0.72)\end{array}$ & $\begin{array}{l}1997- \\
2012\end{array}$ & $\begin{array}{c}0.20(-0.33 \\
\text { to } 0.72)\end{array}$ & & & & \\
\hline & $\begin{array}{l}2 \text { year } \\
\text { survival rate }\end{array}$ & $\begin{array}{c}79.4 \%(69.89 \\
\text { to } 86.17)\end{array}$ & $\begin{array}{c}78.6 \%(71.39 \\
\text { to } 84.22)\end{array}$ & $\begin{array}{c}-0.02(-0.67 \\
\text { to } 0.63)\end{array}$ & $\begin{array}{l}1997- \\
2012\end{array}$ & $\begin{array}{c}-0.02(-0.67 \\
\text { to } 0.63)\end{array}$ & & & & \\
\hline & $\begin{array}{l}5 \text { year } \\
\text { survival rate }\end{array}$ & $\begin{array}{c}69.1 \%(58.84 \\
\text { to } 77.25)\end{array}$ & $\begin{array}{l}66.4 \%(57.54 \\
\text { to } 73.84)\end{array}$ & $\begin{array}{c}-0.12(-1.19 \\
\text { to } 0.95)\end{array}$ & $\begin{array}{l}1997- \\
2012\end{array}$ & $\begin{array}{c}-0.12(-1.19 \\
\text { to } 0.95)\end{array}$ & & & & \\
\hline
\end{tabular}

APC, annual percent change; CI, confidence interval; CCI, Charlson Comorbidity Index. $* P<0.05$.

a The last year for the 1-year and 2-year survival rate is 2012; the last year for the 5-year survival rate is 2010.

may not be the true cause behind the epidemic of thyroid cancer in Taiwan, since follicular carcinoma was not the predominant increased subtype in recent years.
Therefore, the recent emergence of environmental carcinogens is the most possible candidate risk factors accounting for the epidemic of thyroid cancer in Taiwan. 
The westernized post-industrial lifestyle has profoundly affected the dietary habits in Taiwan, and the increased nitrite and nitrate ingestion through drinking water contaminated by fertilizers and through processed food has already been associated with an increased risk of thyroid cancer [36]. Besides, residents in volcanic areas are at higher risk of papillary thyroid cancers, suggesting that a volcanic environment with trace elements or heavy metals may play a role in the carcinogenesis of thyroid cancer [37]. These potential thyroid-specific carcinogens, such as solvents, pesticides, plastic constituents, and heavy metals, might interfere with our hormone homeostasis and increase the thyroid susceptibility to environmental carcinogens. For example, Polybrominated diphenyl ethers, a flame retardant commonly used in a variety of textiles and household products, are recognized to alter thyroid hormone homeostasis, but its association with thyroid cancer was not proved in a large-scale case-control trial [38]. Therefore, so far, no solid evidence for thyroidspecific carcinogens or carcinogenesis has been identified.

\section{Treatment strategies for patients with thyroid cancer}

According to the American Joint Committee on Cancer TNM staging for thyroid cancer (7th edition, 2010), thyroid cancers are staged based on the primary tumor size and presence of extra-thyroid extension. For T0 patients (no evidence of primary malignant tumor), no local surgery or radiation treatment is recommended. Thyroid lobectomy alone is recommended for patients with thyroid microcarcinoma, but surgeons often perform total thyroidectomy in most clinical settings (74.3\%), and more than one-fifth of patients reportedly received additional radioiodine ablation in the SEER database [39, 40]. However, thyroid cancer-specific survival analysis showed no survival advantage in those patients with thyroid microcarcinoma receiving surgery or radiation therapy [40]. Lobectomy or total thyroidectomy is the treatment of choice for primary thyroid cancers that measure 1-4 cm in the greatest dimension; however, aggressive total thyroidectomy is preferred, because it can reduce the risks of local recurrence and nodal metastasis. Total thyroidectomy with neck dissection is recommended for tumors larger than $4 \mathrm{~cm}$ in the greatest dimension or for cases of evident locoregional metastasis. Thyroidectomy carries a 1-6\% risks of complications, including hypothyroidism, hypoparathyroidism, and vocal cord paralysis, while lobectomy is associated with complications in roughly half as many risks [41]. The 2015 American Thyroid Association (ATA) management guidelines recommend that thyroid lobectomy alone may be sufficient as the initial treatment for low-risk, unifocal,

A

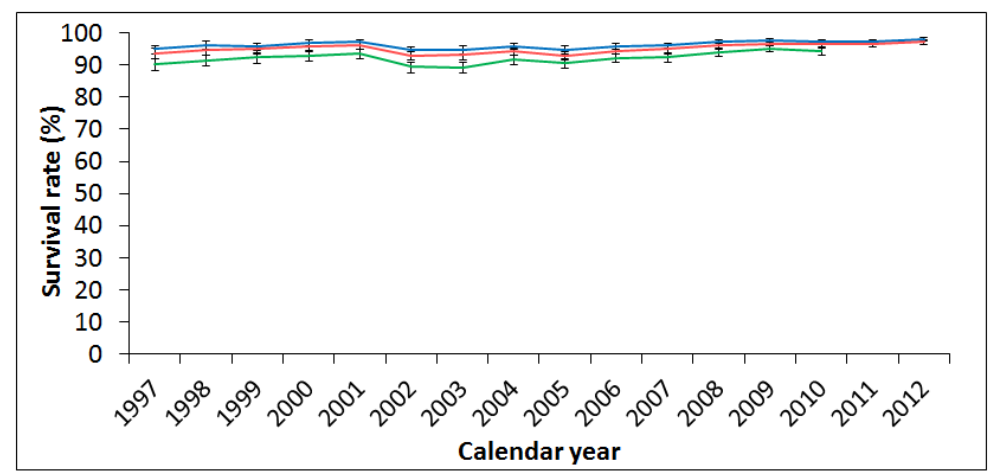

B

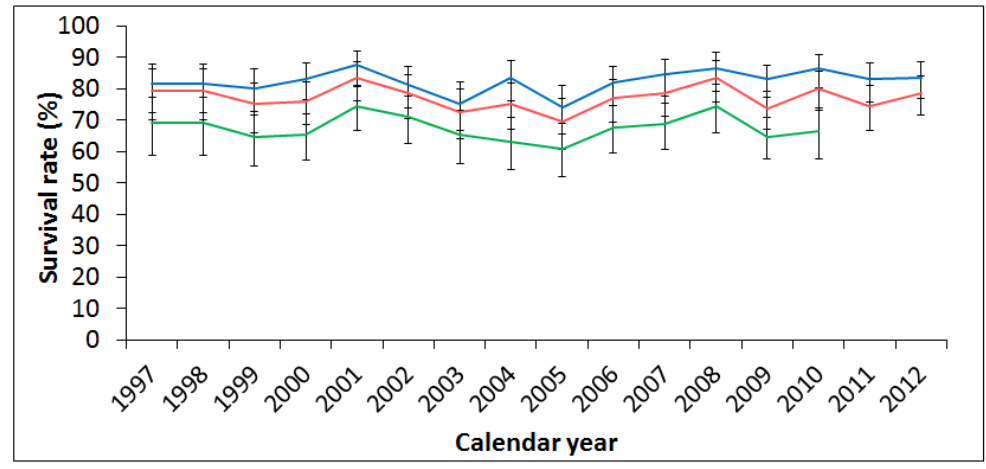

Figure 8: Secular trends of the one-, two-, and five-year survival rates of thyroid cancer in patients with (A) Charlson Comorbidity Index $(\mathrm{CCI}) \leq 3$; (B) CCI $\geq 4$ in Taiwan from 1997 to 2012. (Blue: 1-year survival rate, red: 2-year survival rate, green: 5-year survival rate.) 
and intrathyroidal differentiated thyroid carcinomas $<4 \mathrm{~cm}$, unless there are clear indications to remove the contralateral lobe [42].

Iodine-131 is a unique radioactive iodine that targets thyroid tissue and is widely used to eliminate occult residual tumors in order to reduce the risk of recurrence. Radioactive iodine is also used to treat persistent diseases and to ablate any remnant thyroid tissue, thereby facilitating further surveillance by serum thyroglobulin or radioiodine whole-body scintigraphy [3]. Postoperative adjuvant radioiodine ablation is recommended for $\mathrm{T} 4$ or metastatic patients, but is not recommended for tumors $<1 \mathrm{~cm}$, and is not routinely recommended for tumor $<4$ $\mathrm{cm}$ in diameter after lobectomy or total thyroidectomy in the absence of other adverse features [42]. For lowrisk thyroid cancer, recombinant human thyrotropin and postoperative low-dose $(30 \mathrm{mCi})$ radioiodine ablation may be sufficient for ablating the remnant thyroid [43]. BRAF-mutated cancers and those that are positive on fluorodeoxyglucose positron emission tomography scans are often refractory to radioiodine [3].

Patients with a low to intermediate risk disease are monitored by neck ultrasonography and assessment of their serum thyroglobulin levels [3]. Thyroid cancers are often indolent, even when they have metastasized to distant sites. Currently, systemic therapy is reserved for patients with metastatic disease that is progressing, symptomatic, or threatening vital structures and that is not amenable to localized therapies. Palliative radiotherapy alone, combined radiotherapy and low-dose chemotherapy, or local therapies may control the disease in patients with unresectable regional or metastatic disease. With the advances in molecular genetics, molecular targeted therapy with tyrosine kinase inhibitors such as sorafenib, lenvatinib, vandetanib, and cabozantinib has been introduced for radioiodine-refractory patients to control tumor progression and prolong disease-free survival [3]. In the future, the results of genetic profiling will be incorporated into this stratified process [44]. For example, a mutation in the telomerase reverse transcriptase (TERT) promoter is an independent indicator of poor prognosis for all differentiated thyroid carcinomas [45].

In 2006, the ATA first published their management guidelines for patients with thyroid nodules and differentiated thyroid cancer [46]. In these guidelines, total or near-total thyroidectomy was recommended as the initial surgical procedure for most thyroid cancer patients, and thyroid lobectomy alone was recommended for small $(<1 \mathrm{~cm})$, low-risk, intrathyroidal tumors in the absence of nodal metastases; routine central neck dissection was suggested for patients with papillary thyroid carcinoma; and radioiodine was recommended for patients with stage II-IV disease. The release of the 2006 ATA guidelines changed the clinical management for differentiated thyroid cancer in the US, with significantly more patients receiving total thyroidectomy, lymphadenectomy, and radioiodine after 2006, as disclosed by analysis of the SEER database [47]. In our study, the joinpoint analysis of thyroid cancer treatments showed accelerating trends of total thyroidectomy, neck dissection, and radioiodine ablation accompanied by a decelerating trend of partial thyroidectomy after 2006; these findings may be coincident or be related to the publication of the 2006 ATA guidelines. It is possible that the trends in thyroid cancer management in Taiwan might change again after the 2015 ATA guideline revision, with less use of extensive surgeries and radioiodine ablation, but more utilization of tyrosine kinase inhibitors [48].

\section{Prognostic factors for patients with thyroid cancer}

In our analyses, the prognostic factors for overall survival of thyroid cancer patients were identified as the histological subtype, age at diagnosis, sex, and comorbidities. Among these, the histological subtype was the most significant prognostic factor. In a multivariate analysis of the SEER database during 1988-2007, the reported independent prognostic factors for thyroid cancer-specific survival were as follows: age $>45$ years, aggressive histopathology, advanced tumor stage, tumor size $>4 \mathrm{~cm}$, extrathyroidal extension, lymph node or distant metastasis, and postoperative radiation [49]. Advanced age is associated with poor prognosis in patients with papillary thyroid carcinoma, and a transcriptional profiling study showed that metabolic alterations and immune dysregulation are possible underlying mechanisms [50]. Among these variables to stratify the risks of recurrence, several molecular markers have shown promise. Especially, mutations of the TERT promoter, RET, and BRAF are established as major prognostic biomarkers and are associated with clinical aggressiveness of papillary thyroid carcinoma [51, 52]. In Chinese patients with papillary carcinoma, TERT promoter mutations rather than BRAF V600E mutations were associated with a more advanced TNM stage and shorter progression-free survival [53].

\section{Future aspects for thyroid cancer managements}

The increase in health care expenditures related to over-detection and management of these presumably lowrisk thyroid cancers with possibly unfavorable treatment outcomes has resulted in a backlash trend against the over-detection and over-treatment for thyroid cancer. Reports from Japan comparing active surveillance with immediate surgery for more than 2,000 patients with low-risk papillary microcarcinoma showed equally excellent oncological outcomes and fewer unfavorable events [54]. However, before being confirmed by a largescale prospective study, the active surveillance approach should be considered the preferred option for patients 
with low-risk papillary thyroid carcinomas. Diagnostic risk-stratification can help identify the patients who should be considered for aggressive management or watchful waiting. Future investigations should include evaluations of thyroid cancer molecular prognostication and identification of novel thyroid-specific carcinogens, randomized clinical trials for evaluating the outcome of active surveillance approaches, and studies aimed at identifying better diagnostic criteria to recognize and differentiate subclinical thyroid lesions from progressing and invasive cancers. With time, molecular profiling of fine-needle aspiration specimens or postoperative samples for patients with thyroid cancer will facilitate the development of risk-stratified management and individualized treatment strategies.

\section{Limitations of this study}

There are several limitations to our study. First, the NHI research database contains no clinical information on cancer stage, tumor size and histological subtype, and information about cancer stage and tumor size of thyroid cancer is still unobtainable from the Taiwan Cancer Registry database, not to mention that molecular profiling of thyroid cancer is still not generally performed for thyroid cancer specimens in Taiwan. Therefore, we could not evaluate the associations of thyroid cancer stage and tumor size with their corresponding survival rates, or estimate the magnitude of overdiagnosis by determining the tumor size or stage at diagnosis. However, the other presented available clinical information of thyroid cancer from the NHI database has excellent agreement with those from the Taiwan Cancer Registry. Those data are inherently complementary and are supported by consistent epidemiologic statistics between previous studies and our results.

Second, the treatment procedures for thyroid cancer presented herein included those administered after the diagnosis of thyroid cancer. However, treatments might take place before the diagnosis of thyroid cancer in clinical practice, such as thyroid operations for benign diseases. Therefore, the presented numbers of treatment procedures for thyroid cancer likely underestimate the true situation, especially in terms of the numbers of thyroid lobectomies and total thyroidectomies. Additionally, because of the lack of environmental pollutions and radiation exposure data, we were not able to assess the associations between environmental carcinogens and the incidence of thyroid cancer. Besides, there is a potential for coding errors in the analysis of large national databases, which might possibly result in underestimation of the incidence and treatment procedures of thyroid cancer, even though these nationwide datasets are reported with standardized abstraction methods and are highly scrutinized. Finally, since we obtained information of thyroid cancer patients from various databases, the creation of a risk stratification models of prognostic factors for thyroid cancer-specific survival became impossible, because the eligible populations in these databases differed.

Despite these limitations, this is, to our knowledge, the first large-scale epidemiological study of thyroid cancer in Taiwan combining nationwide data from the NHI database, the Taiwan Cancer Registry, and the National Death Registry, making the reported findings more generalizable for the Taiwanese population. To eliminate the risk of underestimation of the survival rates from the NHI database, we crosslinked the National Death Registry to obtain the most accurate mortality rates. We also provided abundant clinicodemographic information, including information on the patients' socioeconomic status, histological subtypes, comorbidities, and geographic variations, to make our present report more comprehensive and trustworthy.

\section{CONCLUSION}

In this epidemiological study of thyroid cancer in Taiwan during 1997-2012, we found 2.2- and 4.2-fold increases in the incidence and prevalence of thyroid cancer, respectively, especially predominant in the papillary subtype. The female-to-male ratio of agestandardized incidence rates decreased from 4.8 in 1997 to 3.3 in 2012 . The male patients presented at older age at diagnosis and had worse prognosis than female cases. Thyroid cancer was more prevalent in patients with high socioeconomic status and this disparity grew even more pronounced in recent years. The overall survival rates by sex and subtype remained stable over time, with 5-year survival rates of $90.2 \%$ in 1997 and $92.4 \%$ in 2010 . Taken together, the rising incidence (predominant in the papillary subtype) but stable survival rates, and the marked increase in high socioeconomic status, imply that enhanced detection of subclinical lesions is the major contributor for the epidemic of thyroid cancer in Taiwan, possibly due to improved diagnostic modalities and increasing medical surveillance. A true increase of thyroid cancer due to thyroid-specific environmental carcinogens might also play some role, but warrant further investigations.

\section{MATERIALS AND METHODS}

\section{Ethics statement}

This epidemiologic study was approved by the institutional review board of Chang Gung Memorial Hospital (approval number: 201601563B1). As the data used in this study were anonymized, the need for patient consent was waived.

\section{Data sources and study population}

Thyroid cancer cases were identified from the Taiwan NHI Research Database between 1997 and 2012. 
This database routinely collects health information from all Taiwan NHI beneficiaries since 1995. The NHI is a single medical expense payer system that covered over $99.6 \%$ of beneficiaries registered in the database at the end of 2014. The NHI research database includes demographic data and information regarding the diagnoses, operations, and prescriptions from both primary and specialist care providers. The personal information in the NHI database during the study period was encrypted and encoded using the International Classification of Diseases, Ninth Revision, Clinical Modification (ICD-9-CM). The validity, representativeness, and clinical consistency of this database have been reported in many previous studies. We also crosslinked the Taiwan Cancer Registry database to gain information about the histological subtypes of thyroid cancer. The survival rates of thyroid cancer patients were obtained from the National Death Registry database.

\section{Case definition of thyroid cancer}

The thyroid cancer cases were identified by using the ICD-9-CM code 193 in the Catastrophic Illness Registry of the NHI database between 1997 and 2012. The Catastrophic Illness Registry is a medical co-payment waiver provided by the Taiwan government in order to reduce the economic burden of patients with severe diseases, including thyroid cancer. Medical professionals should provide pathological or cytological reports to expert panels before approval of waivers. The associated clinical information including the unique personal identification, diagnosis, demographics, application date, diagnosing physician, hospital, and other administration data, is sent to the insurance administration.

\section{Estimation of the prevalence, incidence, and survival rate}

The crude prevalence rate of thyroid cancer per 100,000 individuals was calculated by using the number of prevalent cases divided by the eligible population in that specified calendar year. We defined thyroid cancer patients as individuals who had a medical record of thyroid cancer before July 1 of each calendar year. The denominator for the prevalence estimation was defined as all individuals registered on July 1 of each calendar year. The crude incidence rate of thyroid cancer per 100,000 person-years was calculated by using the number of incident cases divided by the total person-years in an at-risk population accumulated during that same year. Patients with no diagnosis of thyroid cancer prior to January 1 of each calendar year and with a record of thyroid cancer during that year were defined as incident cases. People with no history of thyroid cancer for each calendar year during the same year were defined as our at-risk cohorts. The followup was calculated from January 1 of the year of the earliest date of thyroid cancer diagnosis until death or December
31 of that specified year. To diminish the restrictions of the databases, we only included patients with at least a one-year registration period prior to January 1 of each calendar year in our eligible cohorts. The age-standardized prevalence and incidence of thyroid cancer were calculated in each calendar year between 1997 and 2012, with the population structure in 2012 as the reference. Data about histological subtypes were obtained from the Taiwan Cancer Registry database. Subgroup analyses of prevalence and incidence were conducted according to sex, calendar years, and histological subtypes. The mortality rates of thyroid cancer patients were obtained from the National Death Registry. We estimated the one-year, two-year, and five-year survival rates in the different calendar years using the life table method. We also calculated the geographic variations in prevalence and incidence of thyroid cancer in 1997 and in 2012 by dividing Taiwan into 21 cities and counties, including Taipei city, New Taipei City, Keelung city, Taoyuan county, Hsinchu city and county, Miaoli county, Taichung city, Taichung county, Changhua county, Yunlin county, Nantou county, Chiayi city and county, Tainan county, Tainan city, Kaohsiung county, Kaohsiung city, Pingtung county, Yilan county, Hualien county, Taitung county, and offshore islets (Penghu). To eliminate the effects of different age and sex structures in various regions, we estimated the age-standardized prevalence and incidence of thyroid cancer with respect to the overall population structure of 2012.

\section{Estimation of treatment procedures for thyroid cancer}

We defined five different treatment procedures for thyroid cancer using the ICD-9-CM diagnosis or procedure codes, including partial thyroidectomy (procedure codes: 062, 063), total thyroidectomy (procedure code: 064), lymph node dissection (procedure codes: 403, 404, 405), chemotherapy (diagnosis codes: V581, V073), and external beam radiotherapy (diagnosis codes: V580, V661, V671). We used the codes of the NHI payment system to define patients receiving I-131 ablation therapy. Subsequently, the number of different treatment procedures performed after the diagnosis of thyroid cancer in that specific calendar year were calculated and the average APCs of the treatments were compared.

\section{Statistical analysis}

We estimated the $95 \%$ confidence intervals for the prevalence and incidence of thyroid cancer under the assumption of a Poisson distribution. The Joinpoint Regression Analysis program (version 4.0.4) was used to estimate trends for the prevalence, incidence, survival rates, and the number of treatments of thyroid cancer. This program utilizes the Bayesian Information Criterion to 
generate different numbers of 'joinpoints' when the linear trend of the prevalence, incidence, survival rate, or number of treatments of thyroid cancer changes significantly and calculates the average APC for each segment [55]. The secular trends for survival rates were calculated for patients with a CCI score of $0-3$ vs. $\geq 4$. The CCI score, composed of 17 diagnostic criteria, is often used to evaluate patients' medical burden and to estimate the risk of death. The statistical significance level was set at 0.05 . All statistical analyses were conducted using SAS statistical software (version 9.4; SAS Institute, Cary, NC, USA).

\section{Abbreviations}

National Health Insurance (NHI); annual percentage change (APC); Charlson Comorbidity Index (CCI); International Classification of Diseases, Ninth Revision, Clinical Modification (ICD-9-CM); Surveillance, Epidemiology, and End Results (SEER); American Thyroid Association (ATA); telomerase reverse transcriptase (TERT).

\section{Author contributions}

Conceived and designed: F-C Liu, H-T Lin, H-P Yu; Data acquisition: C-F Kuo, H-P Yu; Data analysis and interpretation: H-T Lin, F-C Liu, S-F Lin, C-F Kuo, T-T Chung; Funding acquisition: F-C Liu, C-F Kuo, H-P Yu; Wrote draft and critical revision: H-T Lin, F-C Liu, S-F Lin, T-T Chung, H-P Yu; Final approval of manuscript: all authors.

\section{ACKNOWLEDGMENTS}

This study was partially based on data from the NHI research database provided by the Bureau of National Health Insurance, Department of Health and Welfare, and managed by the National Health Research Institutes. The sponsors of the study, the Chang Gung Memorial Hospital, the National Science Council, and the University of Nottingham, had no role in the design and conduct of the study; collection, management, analysis, and interpretation of the data; or preparation, review, approval of the manuscript, or decision to submit the manuscript for publication.

\section{CONFLICTS OF INTEREST}

The authors declare no conflicts of interest.

\section{FUNDING}

This work was partially supported by grants from the National Science Council of Taiwan (105-2314-B-182A137-MY3 and 105-2314-B-182A-012-MY3) and the Chang Gung Memorial Hospital (CORP3E0132, CORP3E0152, CMRPG3F1012 and CMRPG3D1671).

\section{REFERENCES}

1. Vigneri R, Malandrino P, Vigneri P. The changing epidemiology of thyroid cancer: why is incidence increasing? Curr Opin Oncol. 2015; 27:1-7.

2. Ferlay J, Soerjomataram I, Dikshit R, Eser S, Mathers C, Rebelo M, Parkin DM, Forman D, Bray F. Cancer incidence and mortality worldwide: sources, methods and major patterns in GLOBOCAN 2012. Int J Cancer. 2015; 136:E359-E386.

3. Fagin JA, Longo DL, Wells SA. Biologic and clinical perspectives on thyroid cancer. N Eng J Med. 2016; 375:1054-1067.

4. Cecoli F, Ceresola EM, Altrinetti V, Cabria M, Cappagli M, Montepagani A, Cuttica CM, Filippi U, Saverino D, Raffa M, Caputo M, Minuto F, Giusti M, et al. Therapeutic strategies and clinical outcome in papillary thyroid microcarcinoma: a multicenter observational study. Eur Thyroid J. 2016; 5:180-186.

5. Randle RW, Balentine CJ, Leverson GE, Havlena JA, Sippel RS, Schneider DF, Pitt SC. Trends in the presentation, treatment, and survival of patients with medullary thyroid cancer over the past 30 years. Surgery. 2017; 161:137-146.

6. O'Grady TJ, Gates MA, Boscoe FP. Thyroid cancer incidence attributable to overdiagnosis in the United States 1981-2011. Int J Cancer. 2015; 137:2664-2673.

7. Chiang CJ, Chen YC, Chen CJ, You SL, Lai MS. Cancer trends in Taiwan. Jpn J Clin Oncol. 2010; 40:897-904.

8. Chiang CJ, Lo WC, Yang YW, You SL, Chen CJ, Lai MS. Incidence and survival of adult cancer patients in Taiwan, 2002-2012. J Formos Med Assoc. 2016; 115:1076-1088.

9. Mao Y, Xing M. Recent incidences and differential trends of thyroid cancer in the USA. Endocr Relat Cancer. 2016; 23:313-322.

10. Aschebrook-Kilfoy B, Grogan RH, Ward MH, Kaplan E, Devesa SS. Follicular thyroid cancer incidence patterns in the United States, 1980-2009. Thyroid. 2013; 23:1015-1021.

11. Safavi A, Azizi F, Jafari R, Chaibakhsh S, Safavi AA. Thyroid cancer epidemiology in Iran: a time trend study. Asian Pac J Cancer Prev. 2016; 17:407-412.

12. Wiltshire JJ, Drake TM, Uttley L, Balasubramanian SP. Systematic review of trends in the incidence rates of thyroid cancer. Thyroid. 2016; 26:1541-1552.

13. Li N, Du XL, Reitzel LR, Xu L, Sturgis EM. Impact of enhanced detection on the increase in thyroid cancer incidence in the United States: review of incidence trends by socioeconomic status within the surveillance, epidemiology, and end results registry, 1980-2008. Thyroid. 2013; 23:103-110.

14. Morris LG, Sikora AG, Tosteson TD, Davies L. The increasing incidence of thyroid cancer: the influence of access to care. Thyroid. 2013; 23:885-891. 
15. Keegan TH, Grogan RH, Parsons HM, Tao L, White MG, Onel K, Horn-Ross PL. Sociodemographic disparities in differentiated thyroid cancer survival among adolescents and young adults in California. Thyroid. 2015; 25:635-648.

16. Guay B, Johnson-Obaseki S, McDonald JT, Connell C, Corsten M. Incidence of differentiated thyroid cancer by socioeconomic status and urban residence: Canada 19912006. Thyroid. 2014; 24:552-555.

17. Siu S, McDonald JT, Rajaraman M, Franklin J, Paul T, Rachinsky I, Morrison D, Imran SA, Burrell S, Hart $\mathrm{R}$, Driedger A, Badreddine $\mathrm{M}$, Yoo J, et al. Is lower socioeconomic status associated with more advanced thyroid cancer stage at presentation? A study in two Canadian centers. Thyroid. 2014; 24:545-551.

18. La Vecchia C, Malvezzi M, Bosetti C, Garavello W, Bertuccio P, Levi F, Negri E. Thyroid cancer mortality and incidence: a global overview. Int J Cancer. 2015; 136:2187-2195.

19. Udelsman R, Zhang Y. The epidemic of thyroid cancer in the United States: the role of endocrinologists and ultrasounds. Thyroid. 2014; 24:472-479.

20. Ahn HS, Kim H, Welch HG. Korea's thyroid-cancer "epidemic"--screening and overdiagnosis. N Engl J Med. 2014; 371:1765-1767.

21. Vaccarella S, Franceschi S, Bray F, Wild C, Plummer M, Dal Maso L. Worldwide thyroid-cancer epidemic? The increasing impact of overdiagnosis. N Engl J Med. 2016; 375:614-617.

22. Park S, Oh CM, Cho H, Lee JY, Jung KW, Jun JK, Won YJ, Kong HJ, Choi KS, Lee YJ, Lee JS. Association between screening and the thyroid cancer "epidemic" in South Korea: evidence from a nationwide study. BMJ. 2016; 355:i5745.

23. Vaccarella S, Dal Maso L, Laversanne M, Bray F, Plummer $\mathrm{M}$, Franceschi S. The impact of diagnostic changes on the rise in thyroid cancer incidence: a population-based study in selected high-resource countries. Thyroid. 2015; 25:1127-1136.

24. Stansifer KJ, Guynan JF, Wachal BM, Smith RB. Modifiable risk factors and thyroid cancer. Otolaryngol Head Neck Surg. 2015; 152:432-437.

25. Wang X, Cheng W, Li J, Zhu J. A meta-analysis of alcohol consumption and thyroid cancer risk. Oncotarget. 2016; 7:55912-55923. https://doi.org/10.18632/oncotarget.10352.

26. Chen Y, Wu F, Saito E, Lin Y, Song M, Luu HN, Gupta PC, Sawada N, Tamakoshi A, Shu XO, Koh WP, Xiang YB, Tomata Y, et al. Association between type 2 diabetes and risk of cancer mortality: a pooled analysis of over 771,000 individuals in the Asia Cohort Consortium. Diabetologia. 2017; 60:1022-1032. doi: 10.1007/s00125-017-4229-z.

27. Kitahara CM, McCullough ML, Franceschi S, Rinaldi S, Wolk A, Neta G, Olov Adami H, Anderson K, Andreotti G, Beane Freeman LE, Bernstein L, Buring JE, ClavelChapelon F, et al. Anthropometric factors and thyroid cancer risk by histological subtype: pooled analysis of 22 prospective studies. Thyroid. 2016; 26:306-318.

28. Jung CK, Little MP, Lubin JH, Brenner AV, Wells SA, Sigurdson AJ, Nikiforov YE. The increase in thyroid cancer incidence during the last four decades is accompanied by a high frequency of BRAF mutations and a sharp increase in RAS mutations. J Clin Endocrinol Metab. 2014; 99:E276-E285.

29. Su X, Li Z, He C, Chen W, Fu X, Yang A. Radiation exposure, young age, and female gender are associated with high prevalence of RET/PTC1 and RET/PTC3 in papillary thyroid cancer: a meta-analysis. Oncotarget. 2016; 7:1671616730. https://doi.org/10.18632/oncotarget.7574.

30. Suzuki S, Suzuki S, Fukushima T, Midorikawa S, Shimura H, Matsuzuka T, Ishikawa T, Takahashi H, Ohtsuru A, Sakai A, Hosoya M, Yasumura S, Nollet KE, et al. Comprehensive survey results of childhood thyroid ultrasound examinations in Fukushima in the first four years after the Fukushima Daiichi nuclear power plant accident. Thyroid. 2016; 26:843-851.

31. Tsuda T, Tokinobu A, Yamamoto E, Suzuki E. Thyroid cancer detection by ultrasound among residents ages 18 years and younger in Fukushima, Japan: 2011 to 2014. Epidemiology. 2016; 27:316-322.

32. Wang SI, Yaung CL, Lee LT, Chiou SJ. Cancer incidence in the vicinity of nuclear power plants in Taiwan: a population-based study. Environ Sci Pollut Res Int. 2016; 23:571-580.

33. Chen TY, Hsu CC, Feng IJ, Wang JJ, Su SB, Guo HR, Huang CC, Lin HJ. Higher risk for thyroid diseases in physicians than in the general population: a Taiwan nationwide population-based secondary analysis study. QJM. 2017; 110:163-168.

34. Teng CJ, Hu YW, Chen SC, Yeh CM, Chiang HL, Chen TJ, Liu CJ. Use of radioactive iodine for thyroid cancer and risk of second primary malignancy: a nationwide populationbased study. J Natl Cancer Inst. 2016; 108:djv314.

35. Tang KT, Wang FF, Pan WH, Lin JD, Won GS, Chau WK, Lin HD, Hsieh YT. Iodine status of adults in Taiwan 2005-2008, 5 years after the cessation of mandatory salt iodization. J Formos Med Assoc. 2016; 115:645-651.

36. Aschebrook-Kilfoy B, Shu XO, Gao YT, Ji BT, Yang G, Li HL, Rothman N, Chow WH, Zheng W, Ward MH. Thyroid cancer risk and dietary nitrate and nitrite intake in the Shanghai women's health study. Int J Cancer. 2013; 132:897-904.

37. Malandrino P, Scollo C, Marturano I, Russo M, Tavarelli M, Attard M, Richiusa P, Violi MA, Dardanoni G, Vigneri R, Pellegriti G. Descriptive epidemiology of human thyroid cancer: experience from a regional registry and the "volcanic factor". Front Endocrinol (Lausanne). 2013; 4:65.

38. Aschebrook-Kilfoy B, DellaValle CT, Purdue M, Kim C, Zhang Y, Sjodin A, Ward MH. Polybrominated diphenyl 
ethers and thyroid cancer risk in the Prostate, Colorectal, Lung, and Ovarian Cancer Screening Trial cohort. Am J Epidemiol. 2015; 181:883-888.

39. Anderson KL Jr, Youngwirth LM, Scheri RP, Stang MT, Roman SA, Sosa JA. T1a versus T1b differentiated thyroid cancers: do we need to make the distinction? Thyroid. 2016; 26:1046-1052.

40. Zhou P, Tian S, Li J, Zhao Y, Liu W, Zhang Y, Hu Z. Paradoxes in thyroid carcinoma treatment: analysis of the SEER database 2010-2013. Oncotarget. 2017; 8:345-353. https://doi.org/10.18632/oncotarget.13395.

41. Tufano RP, Noureldine SI, Angelos P. Incidental thyroid nodules and thyroid cancer. JAMA Otolaryngol Head Neck Surg. 2015; 141:566-572.

42. Haugen BR, Alexander EK, Bible KC, Doherty GM, Mandel SJ, Nikiforov YE, Pacini F, Randolph GW, Sawka AM, Schlumberger M, Schuff KG, Sherman SI, Sosa JA, et al. 2015 American Thyroid Association management guidelines for adult patients with thyroid nodules and differentiated thyroid cancer: the American Thyroid Association guidelines task force on thyroid nodules and differentiated thyroid cancer. Thyroid. 2016; 26:1-133.

43. Schlumberger M, Catargi B, Borget I, Deandreis D, Zerdoud S, Bridji B, Bardet S, Leenhardt L, Bastie D, Schvartz C, Vera P, Morel O, Benisvy D, et al. Strategies of radioiodine ablation in patients with low-risk thyroid cancer. N Engl J Med. 2012; 366:1663-1673.

44. Cheng Q, Li X, Acharya CR, Hyslop T, Sosa JA. A novel integrative risk index of papillary thyroid cancer progression combining genomic alterations and clinical factors. Oncotarget. 2017; 8:16690-16703. https://doi. org/10.18632/oncotarget.15128.

45. Melo M, da Rocha AG, Vinagre J, Batista R, Peixoto J, Tavares C, Celestino R, Almeida A, Salgado C, Eloy C, Castro P, Prazeres H, Lima J, et al. TERT promoter mutations are a major indicator of poor outcome in differentiated thyroid carcinomas. J Clin Endocrinol Metab. 2014; 99:E754-E765.

46. Cooper DS, Doherty GM, Haugen BR, Kloos RT, Lee SL, Mandel SJ, Mazzaferri EL, McIver B, Sherman SI, Tuttle RM; American Thyroid Association Guidelines
Taskforces. Management guidelines for patients with thyroid nodules and differentiated thyroid cancer. Thyroid. 2006; 16:109-142.

47. Goffredo P, Roman SA, Sosa JA. Have 2006 ATA practice guidelines affected the treatment of differentiated thyroid cancer in the United States? Thyroid. 2014; 24:463-471.

48. Kim BW, Yousman W, Wong WX, Cheng C, McAninch EA. Less is more: comparing the 2015 and 2009 American Thyroid Association guidelines for thyroid nodules and cancer. Thyroid. 2016; 26:759-764.

49. Nilubol N, Zhang L, Kebebew E. Multivariate analysis of the relationship between male sex, disease-specific survival, and features of tumor aggressiveness in thyroid cancer of follicular cell origin. Thyroid. 2013; 23:695-702.

50. Hsu YC, Liu CL, Yang PS, Tsai CH, Lee JJ, Cheng SP. Interaction of age at diagnosis with transcriptional profiling in papillary thyroid cancer. World J Surg. 2016; 40:2922-2929.

51. Raue F, Frank-Raue K. Thyroid cancer: risk-stratified management and individualized therapy. Clin Cancer Res. 2016; 22:5012-5021.

52. Jin L, Chen E, Dong S, Cai Y, Zhang X, Zhou Y, Zeng R, Yang F, Pan C, Liu Y, Wu W, Xing M, Zhang X, et al. BRAF and TERT promoter mutations in the aggressiveness of papillary thyroid carcinoma: a study of 653 patients. Oncotarget. 2016; 7:18346-18355. https://doi.org/10.18632/ oncotarget. 7811.

53. Sun J, Zhang J, Lu J, Gao J, Ren X, Teng L, Duan H, Lin Y, Li X, Zhang B, Liang Z. BRAF V600E and TERT promoter mutations in papillary thyroid carcinoma in Chinese patients. PLoS One. 2016; 11:e0153319.

54. Oda H, Miyauchi A, Ito Y, Yoshioka K, Nakayama A, Sasai H, Masuoka H, Yabuta T, Fukushima M, Higashiyama T, Kihara M, Kobayashi K, Miya A. Incidences of unfavorable events in the management of low-risk papillary microcarcinoma of the thyroid by active surveillance versus immediate surgery. Thyroid. 2016; 26:150-155.

55. Kim HJ, Fay MP, Feuer EJ, Midthune DN. Permutation tests for joinpoint regression with applications to cancer rates. Stat Med. 2000; 19: 335-351. 